\title{
Interplay of composition, structure, magnetism, and superconductivity in $\mathrm{SmFeAs}_{1-x} \mathrm{P}_{x} \mathrm{O}_{1-y}$
}

\author{
N. D. Zhigadlo, ${ }^{1, *}$ S. Katrych, ${ }^{1}$ M. Bendele, ${ }^{2,3}$ P. J. W. Moll, ${ }^{1}$ M. Tortello, ${ }^{4}$ S. Weyeneth, ${ }^{2}$ V. Yu. Pomjakushin, ${ }^{5}$ J. Kanter, ${ }^{1}$ \\ R. Puzniak, ${ }^{6}$ Z. Bukowski, ${ }^{1}$ H. Keller, ${ }^{2}$ R. S. Gonnelli, ${ }^{4}$ R. Khasanov, ${ }^{3}$ J. Karpinski, ${ }^{1}$ and B. Batlogg ${ }^{1}$ \\ ${ }^{1}$ Laboratory for Solid State Physics, ETH Zurich, CH-8093 Zurich, Switzerland \\ ${ }^{2}$ Physik-Institut der Universität Zürich, Winterthurerstrasse 190, CH-8057 Zürich, Switzerland \\ ${ }^{3}$ Laboratory for Muon Spin Spectroscopy, Paul Scherrer Institute, CH-5232 Villigen PSI, Switzerland \\ ${ }^{4}$ Dipartimento di Fisica, Politecnico di Torino, 10129 Torino, Italy \\ ${ }^{5}$ Laboratory for Neutron Scattering, Paul Scherrer Institute, CH-5232 Villigen PSI, Switzerland \\ ${ }^{6}$ Institute of Physics, Polish Academy of Sciences, Aleja Lotników 32/46, PL-02-668 Warsaw, Poland \\ (Received 28 July 2011; revised manuscript received 7 September 2011; published 19 October 2011)
}

\begin{abstract}
Polycrystalline samples and single crystals of $\mathrm{SmFeAs}_{1-x} \mathrm{P}_{x} \mathrm{O}_{1-y}$ were synthesized and grown employing different synthesis methods and annealing conditions. Depending on the phosphorus and oxygen content, the samples are either magnetic or superconducting. In the fully oxygenated compounds, the main impacts of phosphorus substitution are to suppress the Néel temperature $T_{\mathrm{N}}$ of the spin density wave (SDW) state and to strongly reduce the local magnetic field in the SDW state, as deduced from muon spin rotation measurements. On the other hand, the superconducting state is observed in the oxygen-deficient samples only after heat treatment under high pressure. Oxygen deficiency as a result of synthesis at high pressure brings the Sm-O layer closer to the superconducting As/P-Fe-As/P block and provides additional electron transfer. Interestingly, the structural modifications in response to this variation of the electron count are significantly different when phosphorus is partly substituting arsenic. Point contact spectra are well described with two superconducting gaps. Magnetic and resistance measurements on single crystals indicate an in-plane magnetic penetration depth of $\sim 200 \mathrm{~nm}$ and an anisotropy of the upper critical field slope of $\sim 4-5$.
\end{abstract}

DOI: 10.1103/PhysRevB.84.134526

\section{INTRODUCTION}

A renewed interest in high-temperature superconductors (HTS) was generated by the unexpected discoveries of Febased oxypnictide superconducting $\mathrm{LaFePO}\left(T_{\mathrm{c}} \cong 5 \mathrm{~K}\right)^{1}$ and $\operatorname{LaFeAs}(\mathrm{O}, \mathrm{F})\left(T_{\mathrm{c}} \cong 26 \mathrm{~K}\right),{ }^{2}$ followed by the subsequent development of homologous series of the quaternary iron pnictides (1111- $L n \mathrm{Fe} P n \mathrm{O}, L n$ : lanthanide, $P n$ : pnictogen) with $T_{\mathrm{c}}$ values up to $\sim 55 \mathrm{~K}$. Several families of iron pnictides and iron chalcogenides were subsequently discovered (see Refs. 3 and 4 and references therein). The compounds belonging to the 1111 family of Fe-based HTS have a common layered structure composed of an alternating stack of $L n \mathrm{O}$ and $\mathrm{Fe} P n$ layers. In all series, the $\mathrm{Fe} P n$ layer is directly responsible for superconductivity, whereas the perovskite-like $L n O$ layers play the role of a charge supplier. Superconductivity occurs through chemical substitution at different atomic sites of the crystal structure, or by applying an external pressure when the antiferromagnetic state is partially or completely suppressed. The resulting electronic phase diagrams depend on the particular compound. ${ }^{4}$ One of the distinctive features of the 1111 family of Fe-based HTS is the possibility to induce superconductivity by applying "chemical pressure" through a partial substitution of the isovalent smaller P ions for the bigger As. Several theoretical ${ }^{5}$ and experimental ${ }^{6}$ studies have addressed this issue. The consensus is that $\mathrm{P}$ substitution for As does not result in significant changes of the electron density but shows a clear influence on the localization of hybridized states, bandwidth, and the topology of the Fermi surface. Interestingly, having the same number of electrons and holes for any $x$ value $\left(\mathrm{As}_{1-x} \mathrm{P}_{x}\right)$ of various parent compounds, one can reliably tune the magnetic character without changing the charge carrier concentrations. However, very different responses on the $\mathrm{P}$ substitution were observed in a variety of systems. Upon $\mathrm{P}$ substitution, superconductivity appears in $\mathrm{BaFe}_{2} \mathrm{As}_{2},{ }^{7} \mathrm{EuFe}_{2} \mathrm{As}_{2},{ }^{8} \mathrm{CaFe}_{2} \mathrm{As}_{2}, \mathrm{SrFe}_{2} \mathrm{As}_{2},{ }^{9}$ (122 family) and $\mathrm{LaFeAsO}^{6}$ as the tetragonal to orthorhombic structural transition temperature is lowered and the associated spin density wave (SDW) transition is suppressed. Nevertheless, no superconductivity was observed in the $\mathrm{CeFeAs}_{1-x} \mathrm{P}_{x} \mathrm{O}$ system down to $2 \mathrm{~K}^{10}$ We note that the substitution of $\mathrm{Fe}$ by isovalent $\mathrm{Ru}$ leads to superconductivity in 122 materials, ${ }^{11}$ while no superconductivity was observed in $\mathrm{PrFe}_{1-x} \mathrm{Ru}_{x} \mathrm{AsO}$ materials,${ }^{12}$ although the structural/SDW transition was completely suppressed. All together, these observations may provide interesting distinctions between the behavior of 1111 and 122 materials upon isovalent substitution, which require further detail investigations. Among many reported compounds, superconductivity in $\mathrm{LaFePO}^{13}$ and $\mathrm{SmFePO}^{14}$ was shown to depend sensitively on the overall composition and the synthesis conditions. Stoichiometric $\mathrm{LaFePO}$ and $\mathrm{SmFePO}$ are metallic and nonsuperconducting. ${ }^{13,15}$ To date, there is no complete and conclusive proof for the occurrence of superconductivity in the $\mathrm{SmFeAs}_{1-x} \mathrm{P}_{x} \mathrm{O}$ system. In Ref. 15, the authors concluded, based on the temperature dependence of the resistivity, that the superconducting window in $\mathrm{SmFeAs}_{1-x} \mathrm{P}_{x} \mathrm{O}$ is very narrow, only in the range $0.5<x<0.65$, with a maximum $T_{\mathrm{c}}$ of $4.1 \mathrm{~K}$ at the optimal doping $x=0.565$.

The main objectives of this study are twofold. First, we investigated the possibility to induce superconductivity in $\mathrm{SmFeAs}_{1-x} \mathrm{P}_{x} \mathrm{O}$ by applying chemical pressure through the substitution of $\mathrm{P}$ ions for As. Second, we explored the relationships among structure, composition, and superconducting properties in order to elucidate the microscopic structural aspects associated with the macroscopic occurrence of superconductivity. We have found, through an exhaustive set of measurements, that the stoichiometric samples prepared 
at ambient pressure are not superconducting down to $2 \mathrm{~K}$ just as LaFePO. ${ }^{13}$ The superconductivity produced in highpressure synthesized samples is due to induced oxygen deficiency, which strongly affects the intra- and interlayer spacing dimensions and the geometry of the $\mathrm{Fe}(\mathrm{As}, \mathrm{P})_{4}$ and $\mathrm{SmO}_{4}$ tetrahedral units.

\section{EXPERIMENTAL DETAILS}

Polycrystalline samples of the $\mathrm{SmFe}(\mathrm{As}, \mathrm{P}) \mathrm{O}$ system were synthesized at ambient pressure (AP) in evacuated quartz ampoules and under high pressure (HP). Powders of SmAs, SmP, $\mathrm{Fe}_{2} \mathrm{O}_{3}$, and $\mathrm{Fe}$ of high purity $(\geqslant 99.95 \%)$ were weighed according to the stoichiometric ratio, thoroughly ground, and pressed into pellets that were then placed into alumina crucibles with cups. The crucibles were loaded to the quartz ampoules, evacuated, filled with Ar gas ( $0.2 \mathrm{~atm})$, and sealed. The ampoules were slowly heated to $660^{\circ} \mathrm{C}$, where the temperature was maintained for $3 \mathrm{~h}$. Next, the temperature was raised to $1060^{\circ} \mathrm{C}$ and maintained for $100 \mathrm{~h}$, and subsequently the ampoules were quenched in cold water. For the high-pressure synthesis, we used the same starting materials. Mixed multiple variants of the synthesis were used as well. In order to recognize the samples synthesized in various conditions, we introduced abbreviation codes describing the sequence of synthesis. For example, the abbreviation HP + AP + HP means that the sample, which was initially synthesized under high pressure (HP), was resynthesized at ambient pressure (AP), and then was once more synthesized under high pressure (HP). For the growth of single crystals, a $\mathrm{NaCl} / \mathrm{KCl}$ flux was mixed with an equal amount of the precursor (for details see Refs. 16 and 17). All procedures related to the sample preparation were performed in a glove box due to the toxicity of arsenic. In HP synthesis, the sample was enclosed in a boron nitride container and placed inside a graphite heater. In a typical run, a pressure of $3 \mathrm{GPa}$ was applied at room temperature. While keeping the pressure constant, the temperature was ramped up in $1 \mathrm{~h}$ to the maximum value of $\sim 1350^{\circ} \mathrm{C}$ and maintained for $4.5 \mathrm{~h}$, followed by quenching. For crystal growth, the maximum temperature was maintained for $72 \mathrm{~h}$, and then decreased to room temperature in $2 \mathrm{~h}$. Afterward, the pressure was released, and the sample was removed. The $\mathrm{NaCl} / \mathrm{KCl}$ flux was dissolved in water.

Powder X-ray diffraction (XRD) studies were performed at room temperature on a $\mathrm{STOE}$ diffractometer $\left(\mathrm{CuK}_{\alpha 1}\right.$ radiation, $\lambda=1.54056 \AA$ ) equipped with a mini-phase-sensitive detector and a Ge monochromator. Powder patterns were refined with the program FULLPROF. ${ }^{18}$ Single crystals were studied at room temperature on a Bruker x-ray single-crystal diffractometer. Data reduction and numerical absorption correction were performed using the Bruker AXS Inc. software package. ${ }^{19}$ The crystal structure was determined by a direct method and refined on $F^{2}$, employing the SHELXS-97 and SHELXL-97 programs. $^{20}$ The magnetization measurements were carried out with a Quantum Design Magnetic Property Measurement System (MPMS-XL). Four-point resistivity measurements were performed in a 14 Tesla Quantum Design Physical Property Measurement System (PPMS). Micrometersized platinum $(\mathrm{Pt})$ leads were precisely deposited onto a platelike crystal using a focused ion beam (FIB) method without altering the bulk superconducting properties. ${ }^{21}$ Point-contact
Andreev-reflection (PCAR) spectroscopy measurements were performed at the Politecnico di Torino, and the details of the experimental setup were described in previous reports. ${ }^{22}$ Zero-field (ZF) and longitudinal-field (LF) muon spin rotation ( $\mu \mathrm{SR}$ ) experiments were performed at the $\pi \mathrm{M} 3$ beam line at the Paul Scherrer Institute (Switzerland). In the ZF experiments, the muons probe the internal field distribution in the samples, whereas LF experiments provide information about the nature of the internal field, whether it is static or dynamic. Neutron powder diffraction experiments were carried out at the SINQ spallation source of the Paul Scherrer Institute using the high-resolution powder diffractometer for thermal neutrons (HRPT). ${ }^{23}$ Since Sm has a very large absorption cross section (5920 barn), we used a double-wall vanadium container with $9 \mathrm{~mm}$ outer and $8 \mathrm{~mm}$ inner diameters to reduce the absorption effect. Calculated attenuation length amounted to $0.5 \mathrm{~mm}$ for $\lambda=1.494 \AA$, assuming the packing density $2 \mathrm{~g} / \mathrm{cm}^{2}$. The double-wall container gives only $1 \mathrm{~mm}$ of the neutron path, making this neutron experiment doable. The sample was rotated during the measurements in order to minimize aberrations due to a nonuniform packing density. The refinements of the crystal structure were carried out with the program FULLPROF. ${ }^{18}$ The following scattering lengths were used: Sm 0.8-i1.65 fm, Fe $9.45 \mathrm{fm}$, P $5.13 \mathrm{fm}$, As $6.58 \mathrm{fm}$, O $5.803 \mathrm{fm}$.

\section{RESULTS AND DISCUSSION}

\section{A. Ambient-pressure vs high-pressure synthesis}

Already at the beginning of our exploratory synthesis study, we recognized a striking difference in the phase purity and magnetic response of the samples obtained at AP and HP conditions. Phosphorus-substituted SmFeAsO samples synthesized at AP were single phase but nonsuperconducting, whereas the HP prepared samples were less pure but showed some diamagnetic response. This motivated us to undertake a systematic study. Figure 1 depicts XRD patterns of polycrystalline $\mathrm{SmFeAs}_{1-x} \mathrm{P}_{x} \mathrm{O}$ samples with nominal phosphorus contents of $x=0.0,0.5$, and 1.0 synthesized at AP and under $\mathrm{HP}$, and combinations of AP and HP treatments. All peaks in the XRD patterns after AP heat treatment can be indexed based on a tetragonal unit cell $(P 4 / \mathrm{nmm})$ of the $\mathrm{ZrCuSiAs}$-type structure. No superconductivity above $2 \mathrm{~K}$ was observed for those samples. In the next step, the sample with nominal composition of $\mathrm{SmFeAs}_{0.5} \mathrm{P}_{0.5} \mathrm{O}$ was heat treated under $\mathrm{HP}$ $\left(\sim 1350^{\circ} \mathrm{C}, 3 \mathrm{GPa}\right)$. This method produced highly dense samples, while the samples synthesized at ambient pressure were not dense, probably due to the low synthesis temperature $\left(1060^{\circ} \mathrm{C}\right)$. In the XRD pattern of the $\mathrm{SmFeAs}_{0.5} \mathrm{P}_{0.5} \mathrm{O}$ sample (abbreviated as AP + HP in Fig. 1), besides the main peaks belonging to the 1111 phase, a few additional tiny peaks were also evident that could be ascribed to traces of $\mathrm{Sm}_{2} \mathrm{O}_{3}$ and SmAs. Thus, the real composition of the polycrystalline samples after HP treatment deviates slightly from the nominal composition, as we also quantify later in the structure analysis. Surprisingly, the magnetization measurement revealed a magnetic response ascribed to superconductivity with $T_{\mathrm{c}}=$ 10.2 K. Realizing the importance of high-pressure treatment in inducing superconductivity, additional synthesis experiments were performed. 


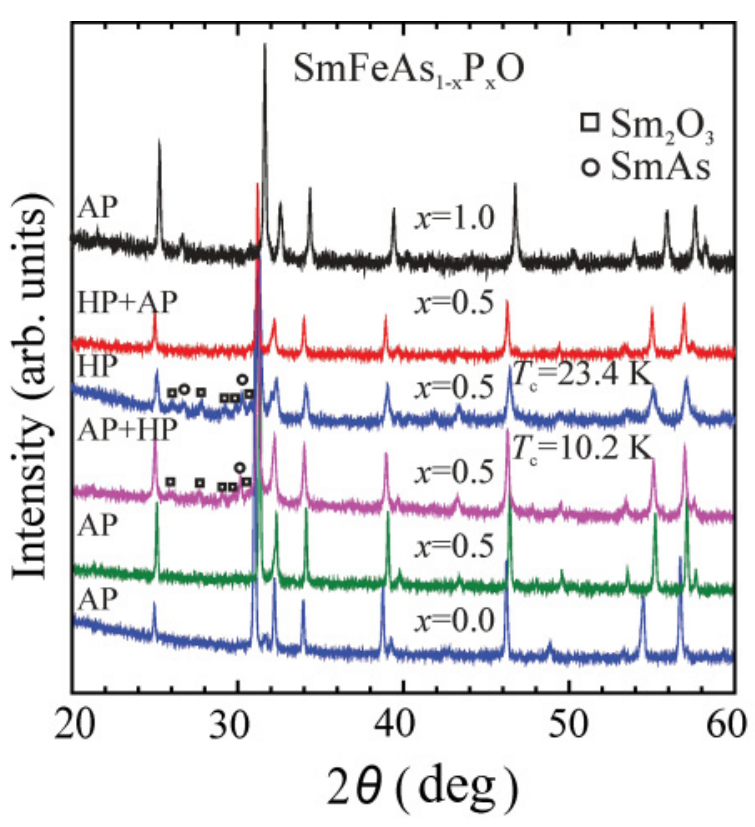

FIG. 1. (Color online) X-ray diffraction patterns of polycrystalline samples with nominal composition $\mathrm{SmFeAs}_{1-x} \mathrm{P}_{x} \mathrm{O}(x=0.0$, 0.5 , and 1.0) synthesized at ambient-pressure (AP) and high-pressure (HP) conditions. The AP sample with $x=0.5$ was heat treated at HP and abbreviated as AP + HP, whereas the HP sample was heat treated at $\mathrm{AP}$ and abbreviated as $\mathrm{HP}+\mathrm{AP}$. The peaks marked by squares and circles belong to $\mathrm{Sm}_{2} \mathrm{O}_{3}$ and $\mathrm{SmAs}$, respectively. The values of $T_{\mathrm{c}}$ were determined from the magnetic susceptibility measurements.

When the $\mathrm{SmFeAs} \mathrm{s}_{0.5} \mathrm{P}_{0.5} \mathrm{O}$ sample was directly synthesized from starting components at HP, superconductivity appeared at $T_{\mathrm{c}, \text { eff }}=23.4 \mathrm{~K}$ [Fig. 2(a)]. XRD revealed in these samples the presence of $\mathrm{Sm}_{2} \mathrm{O}_{3}$ and $\mathrm{SmAs}$ in a slightly higher amount compared to the AP + HP sample (Fig. 1). The left inset in Fig. 2(a) displays the temperature dependence of the electrical resistivity $(\rho)$ of the $\mathrm{SmFeAs}_{0.5} \mathrm{P}_{0.5} \mathrm{O}$ HP sample. The resistivity exhibits metallic character before the onset of the superconducting transition. The room temperature-toresidual resistance ratio is $\sim 3$. The transition width is rather sharp, suggesting a homogeneous nature of the sample.

In the next step, the HP $\operatorname{SmFeAs}_{0.5} \mathrm{P}_{0.5} \mathrm{O}$ sample was sealed in a quartz ampoule and heat treated again in the same manner as was done for the AP synthesized samples. A similar, but opposite effect was observed; i.e., after HP + AP treatment, the samples become practically single phase (Fig. 1, HP + AP) but nonsuperconducting. By again applying high-pressure treatment $(\mathrm{HP}+\mathrm{AP}+\mathrm{HP})$, superconductivity was recovered but at lower $T_{\mathrm{c}}=18.0 \mathrm{~K}$, and the sample had a less amount of impurity phases.

Based on these observations, we conclude that the superconductivity appears only in the nonstoichiometric samples after heat treatment under HP. Phosphorus substitution for As itself does not influence the charge doping, but nonstoichiometry, for example, oxygen deficiency, can introduce electrons into the FeAs/P conducting layer. To check this idea further, we attempted to induce charge carriers through introducing oxygen deficiency. A sample with nominal composition of $\mathrm{SmFeAs}_{0.5} \mathrm{P}_{0.5} \mathrm{O}_{0.85}$ was synthesized in a quartz ampoule in the same way as the previous samples. To avoid/minimize

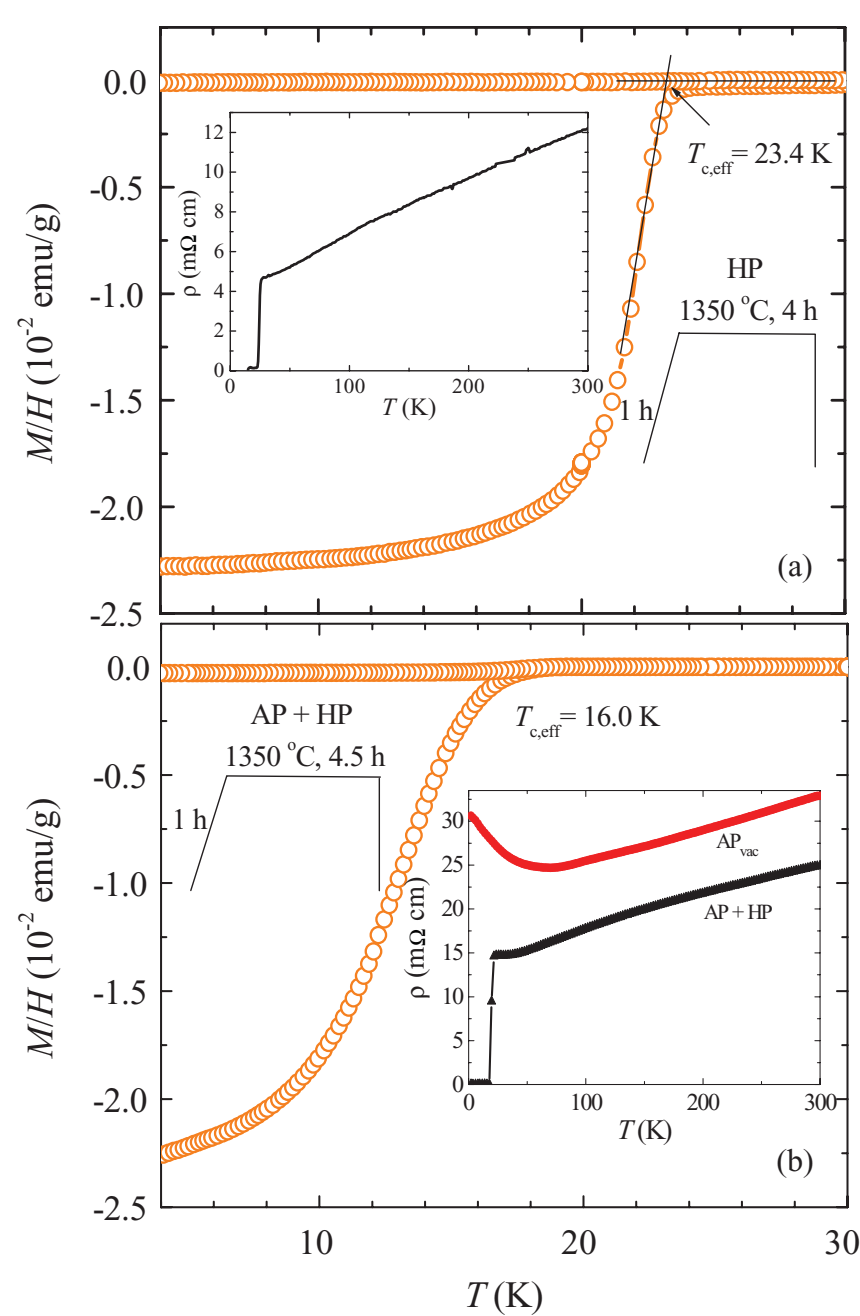

FIG. 2. (Color online) Temperature dependence (zero-fieldcooled and field-cooled) of the magnetic susceptibility of the high-pressure treated superconducting $\mathrm{SmFeAs}_{1-x} \mathrm{P}_{x} \mathrm{O}$ samples. The determination of $T_{\mathrm{c}, \text { eff }}$ is illustrated. In both panels, the temperature history during the high-pressure synthesis processes is sketched. In the insets to Fig. 2(a) and 2(b), the temperature-dependent resistivity for the studied samples is presented.

possible oxygen contamination from the ampoule, the pellet was completely covered by $\mathrm{NaCl}$ powder. No diamagnetism was observed down to $2 \mathrm{~K}$ for this sample. Subsequently, the sample was divided into two parts: One was heat treated at $\mathrm{HP}$, and the second part was sealed, together with a piece of Ta foil, in an evacuated $\left(\sim 10^{-5}\right.$ Torr $)$ quartz ampoule (process abbreviated as $\mathrm{AP}_{\text {vac }}$ ). The HP annealed sample was again superconducting with $T_{\mathrm{c} \text {,eff }}=16.0 \mathrm{~K}$ [Fig. 2(b)] and contained some impurity phases, i.e., $\mathrm{Sm}_{2} \mathrm{O}_{3}$ and SmAs. In contrast, the sample $\mathrm{AP}_{\mathrm{vac}}$, annealed for $3 \mathrm{~h}$ at $1060^{\circ} \mathrm{C}$, was still nonsuperconducting. The right inset in Fig. 2(b) shows the temperature-dependent resistivity for the $\mathrm{AP}_{\mathrm{vac}}$ and $\mathrm{AP}+\mathrm{HP}$ samples. The room-temperature resistivity is larger in $\mathrm{AP}_{\text {vac }}$ than that of AP + HP. No trace of superconductivity was observed in the $\mathrm{AP}_{\mathrm{vac}}$ sample, although the SDW transition was fully suppressed, whereas a superconducting transition at $T_{\mathrm{c}} \sim 18 \mathrm{~K}$ with zero resistivity was observed in the AP $+\mathrm{HP}$ sample. 
Properties of $\mathrm{SmFeAsO}$ samples prepared under $\mathrm{HP}$ conditions were reported in our previous publications. ${ }^{24,25}$ When the HP synthesis starts from stoichiometric SmFeAsO 1111 composition, the superconductivity is not realized, and structural and physical properties are very similar to the SmFeAsO AP samples. Following the results of Ref. 15, we synthesized $\mathrm{SmFeAs}_{0.435} \mathrm{P}_{0.565} \mathrm{O}$ samples at $\mathrm{AP}$ condition, and no superconductivity was detected down to $2 \mathrm{~K}$. Furthermore, we attempted to induce superconductivity in the nonsuperconducting $\mathrm{SmFeAs}{ }_{0.435} \mathrm{P}_{0.565} \mathrm{O}$ sample by applying hydrostatic pressure. This idea was based on our recent observation of pressure-induced superconductivity in $\mathrm{LaFeAsO}_{0.945} \mathrm{~F}_{0.055}{ }^{26}$ In this material, the application of moderate hydrostatic pressure up to $2.4 \mathrm{GPa}$ leads to a substantial decrease of magnetic ordering temperature, reduction of the magnetic phase volume, and, at the same time, a strong increase of the superconducting transition temperature and the diamagnetic susceptibility. Since the magnetic ordering was substantially suppressed by $\mathrm{P}$ substitution (for details, see $\mu \mathrm{SR}$ studies in Sec. D), we expected the possible appearance of superconductivity by application of hydrostatic pressure. For this measurement, a miniature container of $\mathrm{CuBe}$ was employed as a pressure cell, and a mixture of mineral oil and kerosene was used as a pressure-transmitting medium. The pressure at low temperatures was determined by the pressure dependence of the superconducting transition temperature of pure tin placed near the sample. No trace of superconductivity for the $\mathrm{SmFe}_{0.435} \mathrm{P}_{0.565} \mathrm{O}$ sample was found under hydrostatic pressure of $1.3 \mathrm{GPa}$ in the temperature range down to $1.9 \mathrm{~K}$. None of the As-free SmFePO samples showed any indications of superconductivity down to $2 \mathrm{~K}$, whether they were AP and $\mathrm{AP}+\mathrm{HP}$ prepared.

All these results suggest that the superconductivity in the $\mathrm{SmFe}(\mathrm{As}, \mathrm{P}) \mathrm{O}$ system occurs only after a high-pressure treatment, and thus superconductivity may be related to either nonstoichiometry and/or oxygen deficiency. High-pressure preparation may induce sufficient oxygen deficiency and an increased electron concentration sufficient to realize the superconductivity, while annealing in vacuum apparently does not produce enough oxygen deficiency to obtain superconductivity. An even higher oxygen deficiency could lead to decomposition of the compound.

\section{B. Structure modifications due to substitution of $\mathbf{P}$ for As}

The changes of the crystal structure caused by As/P substitution and the structural modifications due to pressureinduced oxygen deficiency were studied by means of x-ray and neutron diffraction. In the P-substituted samples, the XRD peaks shift toward a higher $2 \theta$ value. The (001) peaks shift with $\mathrm{P}$ substitution much more than the (hk0) peaks, indicating that the $c$-axis shrinks more than the $a$-axis (Fig. 1). The lattice constants, atomic position parameters, and selected bond lengths and angles obtained from polycrystalline samples after Rietveld refinement are summarized in Table I. The resulting As/P occupations obtained from $\mathrm{x}$-ray refinement were further confirmed by energy dispersive $\mathrm{x}$-ray spectroscopy (EDX) analysis. For easy comparison, the structural response to $\mathrm{P}$ substitution and to the HP treatment is shown in Fig. 3. It is evident that the changes in the bond length, angle, and layer thickness are significant (see Table I and Fig. 3). The Fe-As distance decreases linearly upon increasing $x$, as expected for $\mathrm{P}$ substitution, ${ }^{27}$ and the As-Fe-As angle $(\alpha)$ increases linearly. Compared to the unsubstituted $\mathrm{SmFeAsO}$ sample $[a=3.92520(4) \AA ; c=8.4693(1) \AA]$, both lattice parameters changed to $a=3.90422(4) \AA$ and $c=8.3206(1) \AA$ for $50 \%$ P substitution (AP method); i.e. $\Delta a / a \approx-0.53 \%$ and $\Delta c / c \approx$ $-1.76 \%$. The unit cell volume difference between $\mathrm{SmFeAsO}$ and $\mathrm{SmFePO}$ is about $5.8 \%$.

The Rietveld refinement of the structural parameters of the SmFeAs ${ }_{1-x} \mathrm{P}_{x} \mathrm{O}$ samples prepared at AP reveals an almost monotonic decrease in the $a$ and $c$ lattice parameters (and unit cell volume) with increasing P substitution. However, the response of the lattice metrics is strongly anisotropic, with the interlayer spacing showing a significantly larger contraction than the change in basal plane dimensions. These values clearly reveal the diversity in bonding, with less contraction in the covalently bonded Fe-As/P layers. The interlayer contraction is $\sim 2.5$ times larger, which is consistent with weaker interlayer interactions. The reduction along the $c$-axis is due to the contraction of the As/P-Fe-As/P layer thickness [S2 in Fig. 3(a)], i.e., a reduction of the FeAs/P height [Fig. 3(c)] when As is replaced by the smaller P, while the $\mathrm{Sm}-\mathrm{As}(\mathrm{P})$ distance and SmO layer thickness remain essentially unchanged with increasing P substitution (Table I).

These trends are different from that in $\mathrm{SmFeAsO}_{1-x} \mathrm{~F}_{x}$, $\mathrm{Sm}_{1-x} \mathrm{Th}_{x} \mathrm{FeAsO}$, and $\mathrm{SmFeAsO}_{1-x},{ }^{24}$ where the $c$-axis lattice constant contraction is due to a large reduction of the $\mathrm{Sm}$-As distance, while the $\mathrm{Sm}-\mathrm{O}$ and As-Fe-As layer thicknesses actually increase with increasing doping. These fundamental differences reflect the fact that $\mathrm{F}$, Th, and $\mathrm{O}$ deficiency brings the Sm-O layer closer to the As-Fe-As block and facilitates electron transfer, while $\mathrm{P}$ substitution in AP samples is a purely geometrical lattice effect without charge carrier transfer. We note that the Fe-As distance $(\sim 2.40 \AA$ in $\mathrm{Sm}-1111$, for example) is essentially doping independent (see $\mathrm{SmFeAsO}_{1-x} \mathrm{~F}_{x}, \mathrm{SmFeAsO}_{1-x}, \mathrm{Sm}_{1-x} \mathrm{Th}_{x} \mathrm{FeAsO}^{24}$ ) but decreases rapidly with increasing $\mathrm{P}$ substitution. The reduced $\mathrm{Fe}-\mathrm{As} / \mathrm{P}$ distance may modify the hybridization between the $\mathrm{Fe} 3 d$ and the As $4 p$ orbitals and thus quench the ordered $\mathrm{Fe}$ magnetic moment.

\section{Structure modifications due to doping by oxygen deficiency}

Next, we discuss some evidence that a variable oxygen content reflects itself in subtle modifications of the structure. As noticed in previous studies ${ }^{28}$ oxygen deficiency in the $L n$-O layer has minute influence on the $L n$-O layer geometry (bond length), but the associated increase in the charge transferred to the Fe- $P n$ layer causes substantial modification of the Fe-Pn bonding geometry. Early theoretical calculations ${ }^{29}$ and recent experimental studies ${ }^{30}$ reveal exceptionally strong dependency of the P-P bond length on the electron count. Remarkably, Fe-P reacts differently from $\mathrm{Fe}-\mathrm{As}$, because of occupation of antibonding P-P states. ${ }^{30}$ Qualitatively, the structure variations in this study appear to follow the same trends. We describe the anomalous changes in the shape of the $\mathrm{Fe}(\mathrm{As}, \mathrm{P})_{4}$ tetrahedra, which are driven by an unusually high sensitivity of the bond length involving $\mathrm{P}$ to the total electron count. 
TABLE I. Refined lattice constants, atomic parameters, and selected bond lengths $(\AA)$ and angles (deg) at room temperature for $\mathrm{SmFeAs}_{1-x} \mathrm{P}_{x} \mathrm{O}$ samples from the Rietveld refinements of the $\mathrm{x}$-ray powder-diffraction profiles. The lattice is tetragonal with space group $P 4 / n m m$. Estimated errors in the last digits are given in parentheses.

\begin{tabular}{|c|c|c|c|c|c|c|c|c|}
\hline Nominal composition & $\mathrm{SmFeAsO}$ & $\mathrm{SmFeAs}_{0.44} \mathrm{P}_{0.56} \mathrm{O}$ & $\mathrm{SmFeAs}_{0.5} \mathrm{P}_{0.5} \mathrm{O}$ & $\mathrm{SmFeAs}_{0.5} \mathrm{P}_{0.5} \mathrm{O}$ & $\mathrm{SmFeAs}_{0.5} \mathrm{P}_{0.5} \mathrm{O}$ & $\mathrm{SmFeAs}_{0.5} \mathrm{P}_{0.5} \mathrm{O}$ & $\mathrm{SmFeAs}_{0.5} \mathrm{P}_{0.5} \mathrm{O}$ & $\mathrm{SmFePO}$ \\
\hline Abbreviation & AP & AP & AP & $\mathrm{AP}+\mathrm{HP}$ & HP & $\mathrm{HP}+\mathrm{AP}$ & $\mathrm{HP}+\mathrm{AP}+\mathrm{HP}$ & AP \\
\hline$T_{\mathrm{c}, \mathrm{eff}}(\mathrm{K})$ & no SC & no SC & no SC & 10.2 & 23.4 & no SC & 18.0 & no SC \\
\hline$a(\AA)$ & $3.92520(4)$ & $3.90225(4)$ & $3.90422(4)$ & $3.90655(6)$ & $3.90664(2)$ & $3.90964(4)$ & $3.9059(1)$ & $3.87431(8)$ \\
\hline$c(\AA)$ & $8.4693(1)$ & $8.3111(1)$ & $8.3206(1)$ & $8.3181(2)$ & $8.3489(4)$ & $8.3369(1)$ & $8.3363(3)$ & $8.1912(2)$ \\
\hline$V\left(\AA^{3}\right)$ & $130.488(3)$ & $126.558(2)$ & $126.831(3)$ & $126.943(4)$ & $127.419(9)$ & $127.431(3)$ & $127.179(7)$ & $122.952(5)$ \\
\hline$z_{\mathrm{Sm}}$ & $0.1371(2)$ & $0.1417(3)$ & $0.1412(3)$ & $0.1418(2)$ & $0.1416(4)$ & $0.1411(2)$ & $0.1416(4)$ & $0.1466(4)$ \\
\hline$B_{\text {iso }}{ }^{\mathrm{a}}\left(\AA^{2}\right)$ & $1.37(9)$ & $2.25(7)$ & $1.58(8)$ & $3.84(8)$ & $2.4(2)$ & $2.23(7)$ & $2.4(2)$ & $1.80(9)$ \\
\hline Fe $B_{\text {iso }}\left(\AA^{2}\right)$ & $2.3(2)$ & $2.62(2)$ & $2.35(18)$ & $5.3(2)$ & $4.1(4)$ & $2.7(2)$ & $4.3(3)$ & $2.02(2)$ \\
\hline$z_{\mathrm{As} / \mathrm{P}}$ & $0.6618(4)$ & $0.6537(7)$ & $0.6537(7)$ & $0.6586(7)$ & $0.658(1)$ & $0.6536(7)$ & $0.656(1)$ & $0.643(2)$ \\
\hline$B_{\text {iso }}\left(\AA^{2}\right)$ & $1.2(2)$ & $2.2(2)$ & $1.6(2)$ & $4.2(2)$ & $3.0(5)$ & $2.3(2)$ & $3.9(4)$ & $2.3(3)$ \\
\hline Occupation As/P & $1.00 / 0.00(2)$ & $0.44 / 0.56(1)$ & $0.50 / 0.50(2)$ & $0.48 / 0.52(1)$ & $0.60 / 0.40(3)$ & $0.52 / 0.48(1)$ & $0.62 / 0.38(2)$ & $0.00 / 1.00(1)$ \\
\hline $\mathrm{O} B_{\text {iso }}\left(\AA^{2}\right)$ & $1.2(7)$ & $3.5(6)$ & $1.2(5)$ & $4.6(6)$ & $1.6(9)$ & $2.9(6)$ & $3.0(9)$ & $3.7(9)$ \\
\hline$R_{\mathrm{p}}(\%)$ & 1.65 & 1.85 & 1.68 & 1.11 & 1.53 & 1.37 & 1.54 & 1.77 \\
\hline$R_{\mathrm{wp}}(\%)$ & 2.17 & 2.35 & 2.17 & 1.51 & 1.92 & 1.73 & 1.94 & 2.33 \\
\hline$R_{B} / R_{F}$ & $3.94 / 4.81$ & $5.28 / 6.21$ & $5.61 / 6.39$ & $7.64 / 6.61$ & $5.11 / 4.48$ & $6.40 / 5.68$ & $5.42 / 4.94$ & $13.4 / 15.4$ \\
\hline Sm-As/P ( $(\AA)$ & $3.256(2)$ & $3.241(3)$ & $3.246(3)$ & $3.223(3)$ & $3.230(5)$ & $3.251(3)$ & $3.236(5)$ & $3.238(8)$ \\
\hline $\mathrm{Sm}-\mathrm{O}(\AA)$ & $2.2804(9)$ & $2.279(1)$ & $2.278(1)$ & $2.2818(9)$ & $2.283(2)$ & $2.2815(9)$ & $2.282(2)$ & $2.279(2)$ \\
\hline $\mathrm{Sm}_{1}-\mathrm{Sm}_{2}(\AA)$ & $3.619(2)$ & $3.628(2)$ & $3.625(2)$ & $3.633(2)$ & $3.636(3)$ & $3.630(2)$ & $3.633(3)$ & $3.643(3)$ \\
\hline $\mathrm{Sm}_{2}-\mathrm{Sm}_{3}(a)(\AA)$ & $3.92520(4)$ & $3.90225(4)$ & $3.90422(4)$ & $3.90655(6)$ & $3.90664(2)$ & $3.90964(4)$ & $3.9059(1)$ & $3.87431(8)$ \\
\hline $\mathrm{Fe}-\mathrm{As} / \mathrm{P}(\AA)$ & $2.394(2)$ & $2.332(3)$ & $2.334(3)$ & $2.357(3)$ & $2.357(6)$ & $2.337(3)$ & $2.347(5)$ & $2.262(8)$ \\
\hline $\mathrm{O}-\mathrm{O}=\mathrm{Fe}-\mathrm{Fe}(\AA)$ & 2.7755 & 2.7593 & 2.7607 & $2.7624(4)$ & 2.7624 & 2.7645 & 2.7619 & 2.7396 \\
\hline $\mathrm{As}_{1}-\mathrm{As}_{2}(\AA)$ & $3.901(3)$ & $3.760(6)$ & $3.764(6)$ & $3.820(6)$ & $3.82(1)$ & $3.769(6)$ & $3.795(9)$ & $3.601(14)$ \\
\hline $\mathrm{As}_{2}-\mathrm{As}_{3}(a)(\AA)$ & $3.92520(4)$ & $3.90225(4)$ & $3.90422(4)$ & $3.90655(6)$ & $3.90664(2)$ & $3.90964(4)$ & $3.9059(1)$ & $3.87431(8)$ \\
\hline $\mathrm{As}_{1}-\mathrm{Fe}-\mathrm{As}_{2}, \beta(\mathrm{deg})$ & $109.13(15)$ & $107.5(2)$ & $107.5(2)$ & 108.3(3) & $108.2(4)$ & $107.5(2)$ & $107.9(4)$ & $105.5(6)$ \\
\hline $\mathrm{As}_{2}-\mathrm{Fe}-\mathrm{As}_{3}, \alpha(\mathrm{deg})$ & $110.15(7)$ & $113.6(1)$ & $113.54(11)$ & $111.93(1)$ & 111.97(19) & $113.54(11)$ & $112.65(18)$ & $117.8(3)$ \\
\hline$S_{3}^{\mathrm{b}}(\AA)$ & $1.703(4)$ & $1.700(6)$ & $1.707(6)$ & $1.660(6)$ & $1.673(8)$ & $1.712(6)$ & $1.687(9)$ & $1.72(2)$ \\
\hline$S_{1}{ }^{\mathrm{b}}(\AA)$ & $2.322(3)$ & $2.355(5)$ & $2.350(5)$ & $2.359(3)$ & $2.364(7)$ & $2.353(3)$ & $2.361(7)$ & $2.402(7)$ \\
\hline$h_{\mathrm{Pn}}{ }^{\mathrm{b}}\left(S_{2} / 2\right)(\AA)$ & $1.370(3)$ & $1.277(6)$ & $1.279(6)$ & $1.319(6)$ & $1.319(8)$ & $1.281(6)$ & $1.300(8)$ & $1.17(2)$ \\
\hline
\end{tabular}

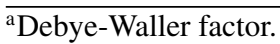

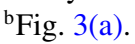

In our $\mathrm{SmFeAs}_{0.5} \mathrm{P}_{0.5} \mathrm{O} \mathrm{HP}$ samples, superconductivity sets in when the $\mathrm{Sm}-\mathrm{O}$ bond length becomes longer $(\sim+0.004$ $0.005 \AA)$ and Sm-As/P becomes shorter $(\sim-0.016-0.023 \AA)$ compared with those in nonsuperconducting $\mathrm{SmFeAs}_{0.5} \mathrm{P}_{0.5} \mathrm{O}$ prepared at AP (Table I). The oxygen deficiency in the samples can be estimated as follows. From the neutron diffraction data of $\mathrm{LaFeAsO}_{1-y}$ and $\mathrm{NdFeAsO}_{1-y}$, a universal relationship between bond length and oxygen deficiency has been established (Fig. 5 in Ref. 28): An increase by $\sim 0.001 \AA$ in Ln-O and a decrease by $\sim 0.04 \AA$ in Ln-As bond lengths corresponds to $\sim 1 \%$ oxygen deficiency. Accordingly, our HP samples contain $\sim 4-6 \%$ O-deficiency. Oxygen deficiency make more electrons available for transfer to the $\mathrm{Fe}-\mathrm{As} / \mathrm{P}$ layer, modifying the bonding geometry in $\mathrm{Fe}(\mathrm{As}, \mathrm{P})_{4}$ and shifting the Fermi level to higher energies. Again, in line with the difference between the Fe-As and Fe-P response to the changing Fermi level, ${ }^{30}$ we notice a similar difference between $\operatorname{SmFeAs}(\mathrm{O}, \mathrm{F})$ and the present compounds with P. As mentioned previously, $\mathrm{FeAs}_{4}$ and $\mathrm{Fe}(\mathrm{As}, \mathrm{P})_{4}$ respond differently to the changes in the electron count. In Ref. 30, the electron count was changed by substitution of $\mathrm{P}$ for $\mathrm{Ge}$, while here we modify it through the chemical composition of the $\mathrm{Sm}-\mathrm{O}$ layer. In $\operatorname{SmFeAs}(\mathrm{O}, \mathrm{F})^{16,17}$ and $(\mathrm{Sm}, \mathrm{Th}) \mathrm{FeAsO},{ }^{24}$ the $\mathrm{FeAs}_{4}$ geometry remained almost unchanged upon F and Th substitution (shaded area in Fig. 4), while in the present study, we found a significant change in the $\mathrm{Fe}(\mathrm{As}, \mathrm{P})_{4}$ geometry upon $\mathrm{O}$ deficiency in the $\mathrm{Sm}-\mathrm{O}$ layer. Figure 3(b) and (c) shows the influence of pressureinduced oxygen deficiency on the interlayer distance $\left(S_{3}\right)$ and pnictogene height $\left(h_{\mathrm{Pn}}\right)$. These structural trends were checked through various heat treatments, and all HP synthesized superconducting samples clearly showed different structural response; i.e., the oxygen deficiency weakens the bonding in the SmO layer, brings it closer to conducting $\mathrm{FeAs} / \mathrm{P}$ layer, and subsequently changes the $\mathrm{Fe}(\mathrm{As} / \mathrm{P})_{4}$ tetrahedron in the direction of $h_{\text {As,P }}$ elongation (Fig. 3).

A direct investigation of the oxygen deficiency is a difficult task. We applied the high-resolution powder neutron diffraction technique to address this issue. AP nonsuperconducting and HP superconducting samples with the same nominal composition of $\mathrm{SmFeAs}_{0.5} \mathrm{P}_{0.5} \mathrm{O}$ were synthesized for neutron diffraction studies. Figure 5 shows a neutron diffraction pattern and its refinement for the AP $\mathrm{SmFeAs}_{0.5} \mathrm{P}_{0.5} \mathrm{O}$ sample. The increase in intensity at large $2 \theta$ is typical for strongly absorbing materials. Fortunately, the structure does not have many parameters, $z_{\mathrm{Sm}}, z_{\mathrm{P}}$, and oxygen occupancy. All atomic displacement parameters (ADP) were fixed by the literature 
(a)

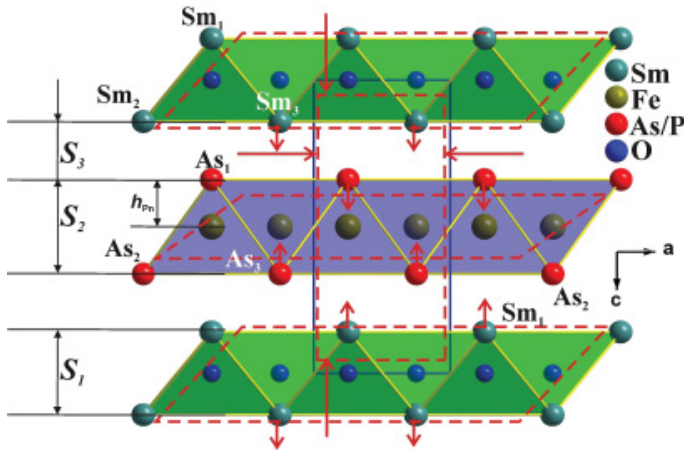

(b)

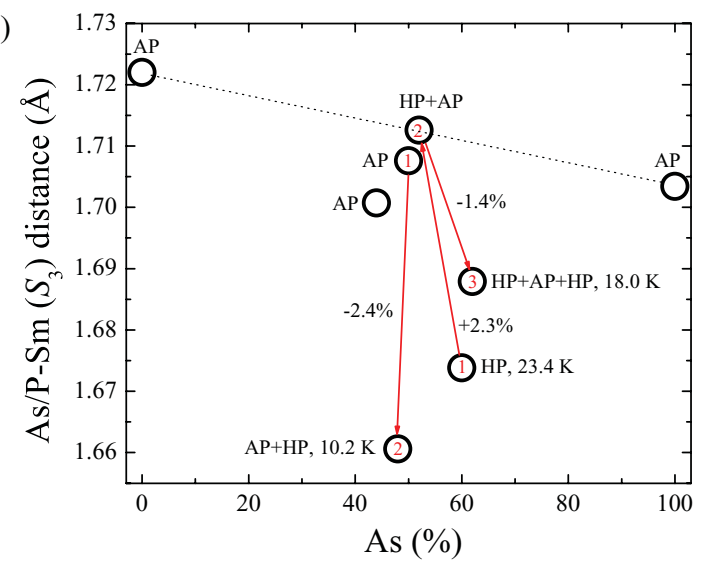

(c)

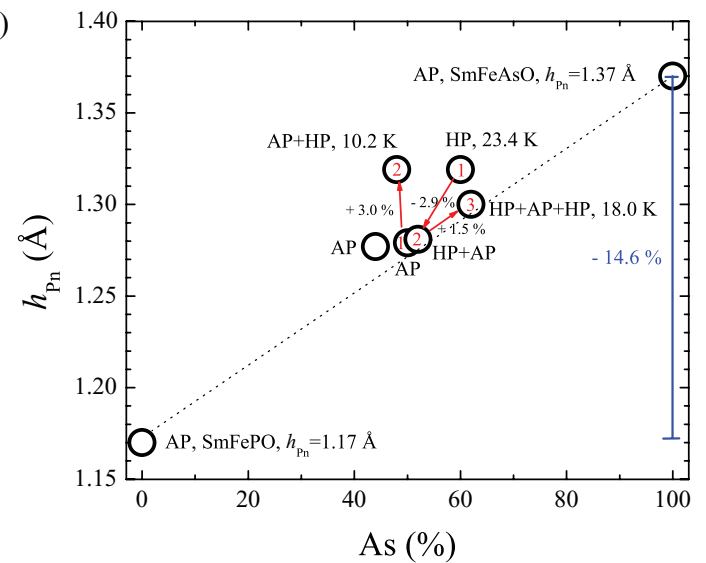

FIG. 3. (Color online) (a) Schematic representation of the projection of the $\mathrm{SmFeAs}_{1-x} \mathrm{P}_{x} \mathrm{O}$ lattice on the $a c$ plane and its changes with substitution of As by $\mathrm{P}$ (red dotted lines). (b) Interlayer distance $S_{3}$ and (c) pnictogen height $h_{\mathrm{Pn}}$ for $\mathrm{SmFe} \mathrm{As}_{1-x} \mathrm{P}_{x} \mathrm{O}$ samples synthesized at ambient pressure (AP), high pressure (HP), and their combinations. Digits in the circles show the sequence of heat treatment. All error bars are smaller than the size of symbols.

data for LaFePO taken from Ref. 13. Both samples were refined with the same varying parameters, namely $z_{\mathrm{P}}$, oxygen occupancy, lattice constants, $U$-half-width parameter of the Cagliotti-type of the resolution function, 11 background Fourier coefficients, overall ADP, and $\sin (2 \theta)$ systematic line shift parameter to account for the absorption. Only $z_{\mathrm{Sm}}$ was fixed, because $\mathrm{Sm}$ has a very small scattering length and does not substantially contribute to scattering. The lattice constant $c=8.33705(63) \AA$ for the HP sample was substantially smaller than that of the AP sample $[c=8.35325(27) \AA]$, whereas $a$ stayed the same within the error bars [HP, $a=$

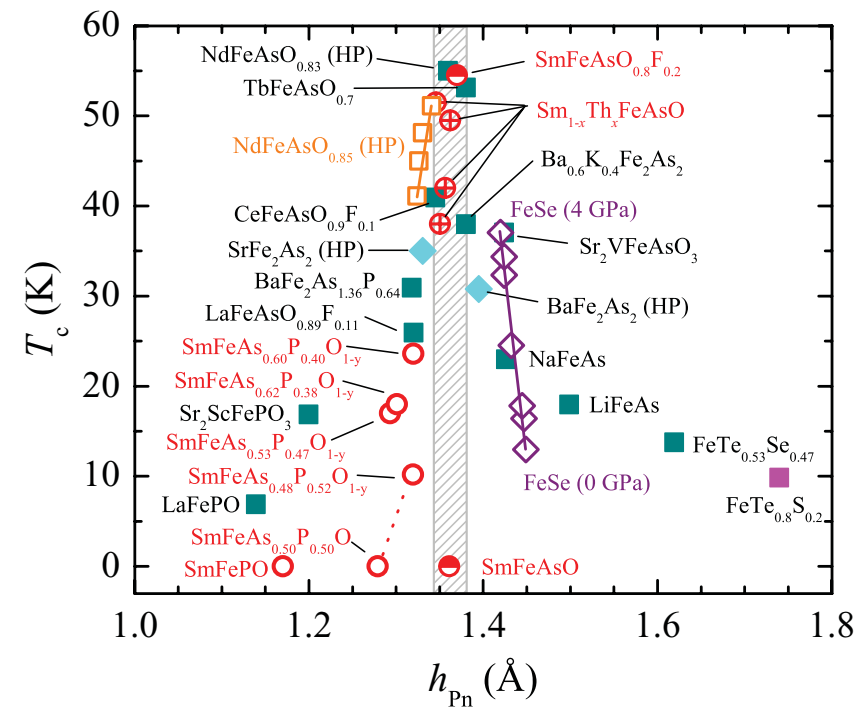

FIG. 4. (Color online) Anion height dependence of the superconducting transition temperature $T_{\mathrm{c}}$ for various Fe-based superconductors, adopted from Ref. 50. Filled squares indicate the data obtained at ambient pressure. Filled diamonds are the $\mathrm{SrFe}_{2} \mathrm{As}_{2}$ and $\mathrm{BaFe}_{2} \mathrm{As}_{2}$ data obtained under optimal pressure. Open squares indicate the $\mathrm{NdFeAsO}_{0.85}$ data under high pressure. The FeSe data under high pressure are indicated by open diamonds. Present experimental results and data from Refs. 16, 17, and 24 are herein indicated by red circles. The dashed line highlights the changes in $h_{\mathrm{Pn}}$ due to oxygen deficiency.

3.91275(30) ̊; AP, $a=3.91244(11) \AA$ A]. However, $z_{\mathrm{As} / \mathrm{P}}$ was larger in the HP sample than that in the AP sample $\left[z_{\mathrm{As} / \mathrm{P}}=0.65397(127)\right.$ for $\mathrm{HP}$ and $z_{\mathrm{As} / \mathrm{P}}=0.64854(65)$ for $\mathrm{AP}]$, resulting in a larger Fe-As/P interplane spacing $\left(h_{\mathrm{As} / \mathrm{P}}\right)$, which is in line with the higher $T_{\mathrm{c}}$. The line broadening was almost two times higher in the HP sample, implying that the sample contained more microstrain (dispersion of lattice constant or inhomogeneity). The sample synthesized under HP clearly showed oxygen deficiency, since its occupancy was smaller than unity [0.85(4)], whereas for AP sample, the

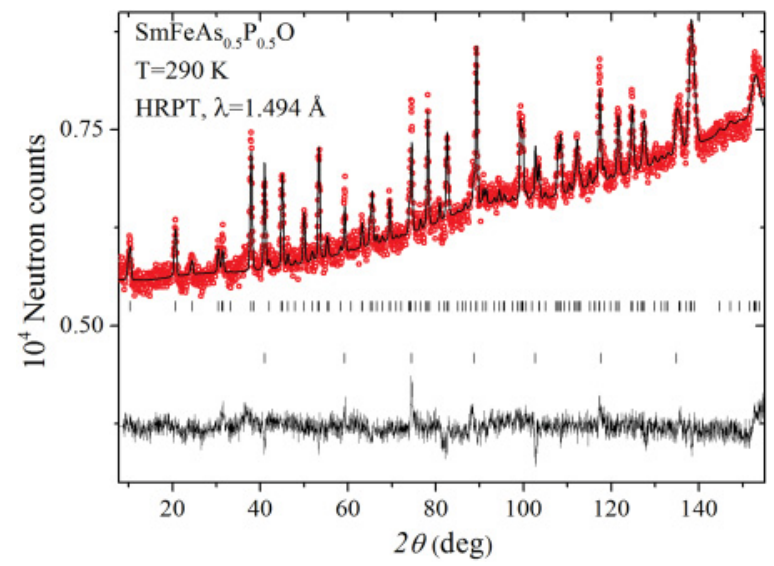

FIG. 5. (Color online) The Rietveld refinement pattern and difference plot of the neutron diffraction data for the $\mathrm{SmFeAs}_{0.5} \mathrm{P}_{0.5} \mathrm{O}$ (AP) sample collected at $290 \mathrm{~K}$ using the instrument with the wavelength $\lambda=1.494 \AA$. The rows of tics show the Bragg peak positions for the main phase and the vanadium sample container. 

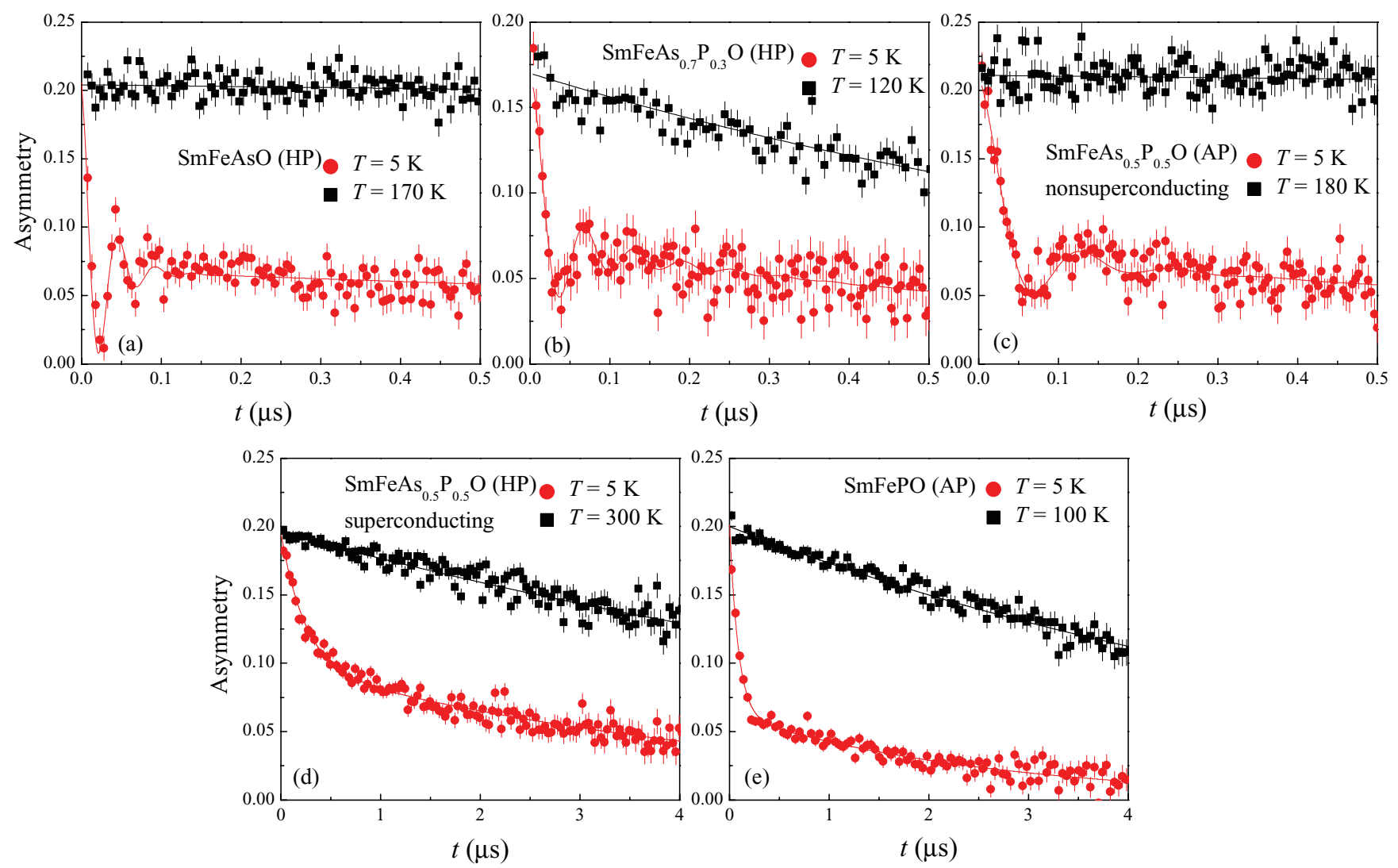

FIG. 6. (Color online) ZF $\mu$ SR time spectra of the muon spin polarization for various dopings of $\mathrm{SmFe}_{\mathrm{As}_{1-x}} \mathrm{P}_{x} \mathrm{O}$ at representative temperatures below and above the magnetic ordering temperature for (a) $\mathrm{SmFeAsO}$ (HP), (b) $\mathrm{SmFeAs}_{0.7} \mathrm{P}_{0.3} \mathrm{O}$ ( $\mathrm{HP}$ ), (c) $\mathrm{SmFeAs}{ }_{0.5} \mathrm{P}_{0.5} \mathrm{O}$ ( $\mathrm{AP}$ ), (d) $\mathrm{SmFeAs}_{0.5} \mathrm{P}_{0.5} \mathrm{O}$ (HP), and (e) $\mathrm{SmFePO}$ (AP).

refined value was $1.07(3)$. With this, we conclude that superconductivity in $\mathrm{SmFeAs}_{1-x} \mathrm{P}_{x} \mathrm{O}$ can be related to the oxygen deficiency.

\section{Muon spin rotation studies: static and dynamic fields}

The polycrystalline $\mathrm{SmFe}(\mathrm{As}, \mathrm{P}) \mathrm{O}$ samples were investigated by muon spin rotation $(\mu \mathrm{SR})$ experiments in zero field (ZF), weak-transverse field (WTF), and longitudinal field (LF). Here, LF and TF denote the cases where the applied magnetic field is parallel and perpendicular to the initial muon spin polarization, respectively. In WTF experiments, the muons stopping in magnetically ordered regions of the samples lost their polarization relatively fast, because the field at the muon stopping site was a superposition of the high internal field and the weak applied external field. For a detailed description of the $\mu$ SR technique, see, e.g., Ref. 31 .

The static or quasistatic magnetic response of the samples was analyzed by using the following functional form: ${ }^{31}$

$$
P^{\mathrm{ZF}}(t)=P_{\text {stat }}\left(\frac{2}{3} f_{\text {osc }} \exp \left[-\lambda_{\mathrm{t}} t\right]+\frac{1}{3} \exp \left[-\lambda_{1} t\right]\right) .
$$

Here, the $2 / 3$ oscillating ( $1 / 3$ nonoscillating) component is caused by the internal fields at the muon stopping site, which are transverse (longitudinal) to the initial muon spin polarization, and $\lambda_{t}$ and $\lambda_{1}$ are the corresponding exponential depolarization rates. The oscillating component takes the form $f_{\text {osc }}=\cos \left(\omega_{0} \mathrm{t}+\phi_{0}\right)$ or $f_{\text {osc }}=J_{0}\left(\omega_{0} \mathrm{t}+\phi_{0}\right)$, depending on the samples. Here, $\omega_{0}$ is the precession frequency, $J_{0}$ is a zeroth- order Bessel function, and $\phi_{0}$ is the phase that is fitted close to zero in all cases. The cosine and the Bessel functions point toward the commensurate and the incommensurate magnetic order, respectively. ${ }^{32}$

As a first step, we describe the $\operatorname{SmFe}(\mathrm{As}, \mathrm{P}) \mathrm{O}$ samples prepared by the HP method. In Fig. 6(a) the oscillating muon time signal of unsubstituted $\mathrm{SmFeAsO}$ reveals that the $\mathrm{SmFeAsO}$ exhibits static commensurate magnetic order, due

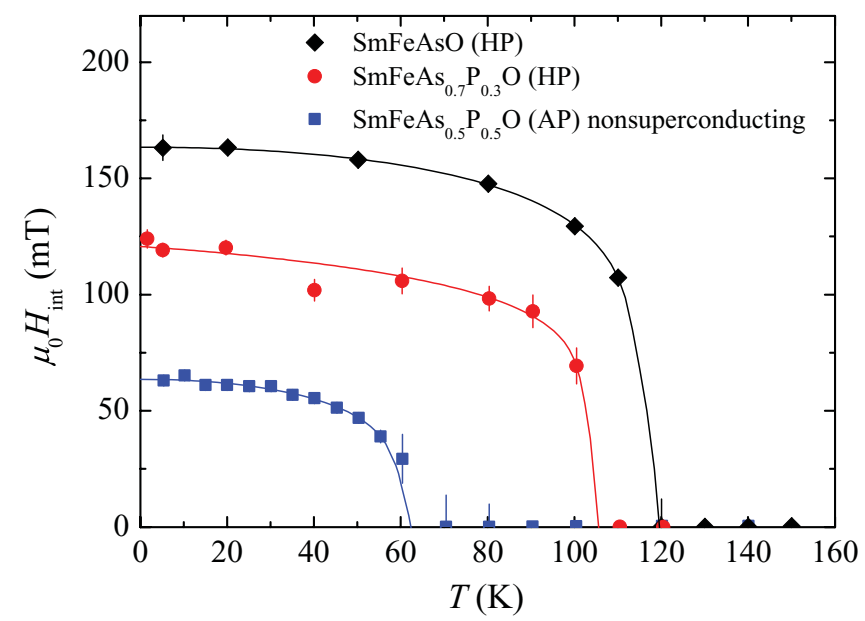

FIG. 7. (Color online) The local field $\mu_{0} H_{\text {int }}$ as a function of substitution and temperature, deduced from the ZF $\mu$ SR measurements on $\mathrm{SmFeAs}_{1-x} \mathrm{P}_{x} \mathrm{O}$. The lines are guides to the eye. 


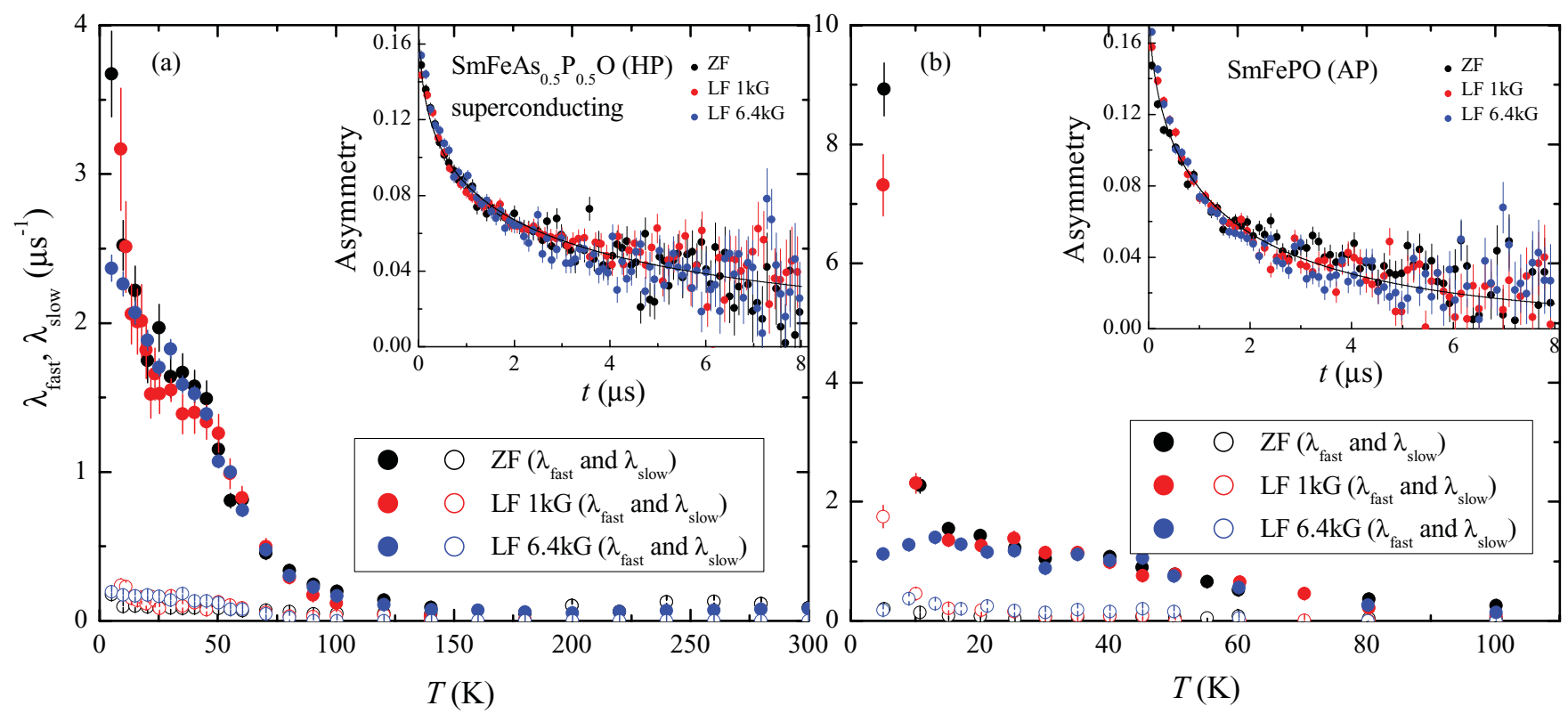

FIG. 8. (Color online) Temperature dependence of the muon depolarization rates $\lambda_{\text {fast }}$ and $\lambda_{\text {slow }}$ of the ZF and LF muon depolarization rates for (a) $\mathrm{SmFeAs}_{0.5} \mathrm{P}_{0.5} \mathrm{O}$ (HP) and (b) $\mathrm{SmFePO}(\mathrm{AP})$. The solid lines represent fits of Eq. (2) to the data.

to the fact that the oscillating part of the muon time signal is described by a simple cosine function. The Néel temperature $T_{\mathrm{N}}=120 \mathrm{~K}$ (see Fig. 7) is reduced compared to earlier measurements of $\mathrm{SmFeAsO}$ synthesized at ambient pressure $\left(T_{\mathrm{N}}=135 \mathrm{~K}^{33}\right)$. Such a difference likely occurs due to slightly reduced oxygen content. As shown previously, this effect could result from the high-pressure synthesis. Upon substituting $30 \%$ of As by $\mathrm{P}$, the sample still exhibits static magnetic order, yet it is described by a Bessel function [Fig. 6(b)]. Both the ordering temperature $T_{\mathrm{N}}$ and the frequency are suppressed compared to the unsubstituted compound (see Fig. 7).

The superconducting $\mathrm{SmFeAs}_{0.5} \mathrm{P}_{0.5} \mathrm{O}$ sample prepared under high pressure shows no oscillations in the $\mathrm{ZF}$ muon time spectra [see Fig. 6(d)]. Instead, a fast decay of the muon spin polarization is observed at low temperature, which could indicate that the magnetism becomes dynamic in the muon time scale in this sample. In fact, evidence for dynamic magnetism follows from LF measurements. The data for both $\mathrm{ZF}$ and LF measurements are best described by a superposition of two exponentially decaying functions. This is in agreement with earlier studies in 1111 compounds and theoretical calculations of the muon stopping site, which revealed two distinct stopping sites of the muons in the 1111 compounds, one close to the Fe atoms in the FeAs/P layers and one near the $\mathrm{LaO}$ planes: ${ }^{34,35}$

$$
P^{\mathrm{ZF}, \mathrm{LF}}(t)=P_{\text {fast }} \exp \left[-\lambda_{\text {fast }} t\right]+P_{\text {slow }} \exp \left[-\lambda_{\text {slow }} t\right] .
$$

Here, $P_{\text {fast }}\left(P_{\text {slow }}\right)$ and $\lambda_{\text {fast }}\left(\lambda_{\text {slow }}\right)$ are the part of the muon spin polarization and the depolarization rate of the fast (slow) relaxing component, respectively. The temperature dependencies of the relaxation rates $\lambda_{\text {fast }}$ and $\lambda_{\text {slow }}$ for both the ZF and LF measurements are shown in Fig. 8(a). Interestingly, up to $\mu_{0} H=0.64 \mathrm{~T}$, both the $\mathrm{ZF}$ and the $\mathrm{LF}$ relaxation rates coincide within almost the whole temperature region. Above $T=80 \mathrm{~K}$, the relaxation rates are only weakly temperature dependent up to $300 \mathrm{~K}$, and the data could be also described by a single exponential decay. Below $T=80 \mathrm{~K}$, both $\lambda_{\text {fast }}$ and $\lambda_{\text {slow }}$ increase with decreasing temperature, whereas they tend to saturate below $T=40 \mathrm{~K}$ to a value of $\lambda_{\text {fast }}=2 \mu \mathrm{s}^{-1}$ and $\lambda_{\text {slow }}=0.2 \mu \mathrm{s}^{-1}$. A further increase in both relaxations is seen again only below $T=10 \mathrm{~K}$.

In $\mathrm{SmFePO}$, the $\mathrm{ZF}$ (and LF) muon time spectra appear similar to those in the superconducting $\mathrm{SmFeAs}_{0.5} \mathrm{P}_{0.5} \mathrm{O}$ sample [see Fig. 6(e)]. Again, the data are best described with Eq. (2). Also the temperature dependence of $\lambda_{\text {fast }}$ and $\lambda_{\text {slow }}$ is very similar to that in $\mathrm{SmFeAs}_{0.5} \mathrm{P}_{0.5} \mathrm{O}$, whereas only the values of $\lambda_{\text {fast }}$ and $\lambda_{\text {slow }}$ are smaller. The fact that both the $\mathrm{ZF}$ and the LF relaxation rates coincide at all temperatures and the exponential character of the muon polarization decay point to the existence of fast electronic fluctuations within the $\mu \mathrm{SR}$ time window for $\mathrm{SmFePO}$ and $\mathrm{SmFeAs}_{0.5} \mathrm{P}_{0.5} \mathrm{O}$ prepared by high-pressure synthesis [Figs. 8(a) and 8(b)]. A similar temperature dependence for $\lambda_{\text {fast }}$ and $\lambda_{\text {slow }}$ was previously observed in superconducting $\mathrm{SmFeAsO}_{0.85}{ }^{36} \mathrm{It}$ was suggested that the fluctuations most probably were not related to superconductivity, ${ }^{36}$ what the presented data confirm, because the fluctuations are present in both the superconducting $\mathrm{SmFeAs}_{0.5} \mathrm{P}_{0.5} \mathrm{O}$ and the nonsuperconducting SmFePO. Furthermore the $T$ dependence for both samples is very similar, and no feature is seen close to $T_{\mathrm{c}}$. The increase of the relaxations below $T=10 \mathrm{~K}$ is most probably associated with additional local field broadening due to the ordering of the Sm moments at low temperatures. ${ }^{36-38}$

However, the muon experiments reveal that the samples prepared initially at low pressure (AP) and those prepared at high pressure and then annealed at ambient pressure $(\mathrm{HP}+$ AP) change their magnetic behavior significantly. This is clearly seen by comparing the $\mathrm{SmFeAs}_{0.5} \mathrm{P}_{0.5} \mathrm{O}$ prepared at high and low pressure. While the HP is superconducting, the AP sample is nonsuperconducting and even shows static magnetic order below $T_{\mathrm{N}}=60 \mathrm{~K}$ (see Fig. 7). Comparison of 
the $\mathrm{ZF}$ muon time spectra shows the difference immediately [Figs. 6(c) and 6(d)]. The HP sample shows a fast decay of the muon spin polarization, pointing to fast fluctuations [Fig. 6(d)], whereas the AP sample shows oscillations indicating static magnetic order [Fig. 6(c)]. Thus, the muon time spectra of the ZF measurements were described again by Eq. (1), whereas the magnetic signal was described by a Bessel function.

The comparison of the two $\mathrm{SmFeAs}_{0.5} \mathrm{P}_{0.5} \mathrm{O}$ samples shows that the preparation procedure [oxygen stoichiometry, geometry of the $\mathrm{Fe}(\mathrm{As}, \mathrm{P})$ layer] influences the electronic properties in an essential way: One method produces superconducting samples with fluctuating magnetism, the other gives nonsuperconducting samples with static magnetic order.

\section{E. Point-contact Andreev-reflection spectroscopy measurements}

Point-contact Andreev-reflection (PCAR) spectroscopy measurements were performed on the HP SmFeAs ${ }_{0.5} \mathrm{P}_{0.5} \mathrm{O}$ sample. PCAR spectroscopy, like scanning tunneling spectroscopy (STS), allows the number, value, and symmetry of the superconducting energy gaps in superconductors to be determined; this is fundamental information necessary to unravel the microscopic mechanism at the origin of superconductivity. However, while STS can be performed only on very flat surfaces of single crystals, PCAR spectroscopy can be reliably used to investigate polycrystalline samples as well.

Instead of using the standard, classical technique, where a sharp metallic tip is pressed against the sample under study, we used the so-called "soft" technique; i.e., the contact is created by means of a small drop of $\mathrm{Ag}$ conducting paste. ${ }^{22}$ In this way, which has proven very useful in studying several materials including Fe-based superconductors, ${ }^{39-41}$ no pressure is applied to the sample, and much more thermally

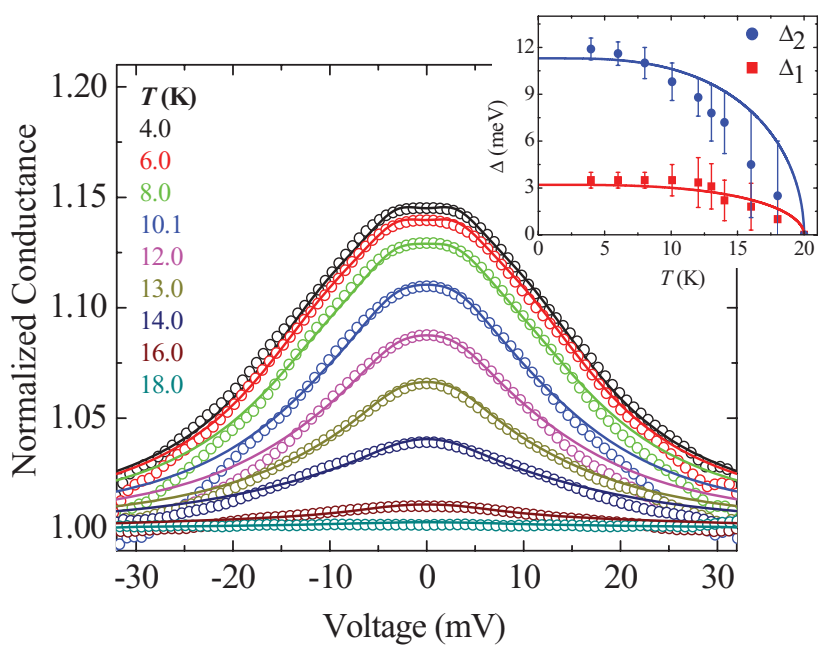

FIG. 9. (Color online) Temperature dependence of the normalized conductance curves (symbols) for the $\mathrm{Ag} / \mathrm{SmFeAs}_{0.5} \mathrm{P}_{0.5} \mathrm{O}$ point contact with their relevant two-band BTK fitting curves (solid lines). The two-band fitting parameters are: $\Delta_{1}=3.5 \mathrm{meV}, \Gamma_{1}=3.4 \mathrm{meV}$, $Z_{1}=0.29, \Delta_{2}=11.9 \mathrm{meV}, \Gamma_{2}=9.15 \mathrm{meV}, Z_{2}=0.225, w_{1}=0.3$. Right inset: temperature behavior of the two gap values (symbols) as obtained by fitting the experimental curves. Two BCS trends are shown for comparison (solid lines). stable contacts are achieved. The experimental conductance curves were obtained by numerical differentiation of the $I-V$ curves, and they have been normalized, i.e., divided by their normal-state curve (at $T=T_{\mathrm{c}}$ ), and fitted to a modified ${ }^{42}$ two-band, $s$-wave Blonder-Tinkham-Klapwijk (BTK) model ${ }^{43}$ generalized to take into account the angular distribution of the current injection at the interface. ${ }^{44}$ In the two-band case, the model is simply the weighted sum of two BTK contributions: ${ }^{41}$

$$
G(E)=w_{1} G_{1}^{\mathrm{BTK}}(E)+\left(1-w_{1}\right) G_{2}^{\mathrm{BTK}}(E),
$$

where $w_{1}$ is the weight of band 1 . Variable $G_{\mathrm{i}}$ is defined by three parameters: the gap value, $\Delta_{i}$, the broadening parameter, $\Gamma_{\mathrm{i}}$, and $Z_{\mathrm{i}}$, which accounts for the height of the potential barrier at the interface and for the mismatch of the Fermi velocities between the normal metal and the superconductor.

Figure 9 shows the temperature dependence of the normalized conductance curves (symbols) for the $\mathrm{Ag} / \mathrm{SmFeAs} \mathrm{S}_{0.5} \mathrm{P}_{0.5} \mathrm{O}$ point contact with the relevant two-band BTK fitting curves (solid lines). The one-gap model is far from reproducing the experimental curves, while a two-gap model fits the experiment remarkably well. From the fit, we get $3.5 \mathrm{meV}$ for the small gap and $11.5-11.9 \mathrm{meV}$ for the larger

TABLE II. Crystallographic and structural refinement parameters for a $\mathrm{SmFeAs}_{0.5} \mathrm{P}_{0.5} \mathrm{O}_{0.85}$ single crystal $(T=295 \mathrm{~K}$, Mo $\mathrm{K} \alpha, \lambda=$ $0.71073 \AA$ ). The absorption correction was done analytically. A fullmatrix least-squares method was employed to optimize $F^{2}$. Some distances and marking of atoms are shown in Figs. 3 and 4.

\begin{tabular}{|c|c|}
\hline Crystallographic formula & $\mathrm{SmFeAs}_{0.53} \mathrm{P}_{0.47} \mathrm{O}_{1-y}$ \\
\hline$T_{\mathrm{c}, \text { eff }}(\mathrm{K})$ & 17 \\
\hline Unit cell dimensions $(\AA)$ & $a=3.91022(8), c=8.3367(2)$ \\
\hline Volume $\left(\AA^{3}\right)$ & $127.467(5)$ \\
\hline$z_{\mathrm{Sm}}($ atomic coordinate $)$ & $0.1414(1)$ \\
\hline$z_{\mathrm{As} / \mathrm{P}}$ & $0.6551(2)$ \\
\hline $\mathrm{Sm}_{1}-\mathrm{Sm}_{2}(\AA)$ & $3.6342(4)$ \\
\hline $\mathrm{O}-\mathrm{O}=\mathrm{Fe}-\mathrm{Fe}(\AA)$ & $2.7649(2)$ \\
\hline $\mathrm{Sm}_{2}-\mathrm{As}_{2}(\AA)$ & $3.2438(7)$ \\
\hline $\mathrm{Sm}-\mathrm{O}(\AA)$ & $2.2831(2)$ \\
\hline $\mathrm{As}_{1}-\mathrm{As}_{2}(\AA)$ & $3.7863(14)$ \\
\hline $\mathrm{Fe}-\mathrm{As} / \mathrm{P}(\AA)$ & $2.3440(7)$ \\
\hline $\mathrm{As}_{1}-\mathrm{Fe}-\mathrm{As}_{2}, \beta(\mathrm{deg})$ & $107.72(3)$ \\
\hline $\mathrm{As}_{2}-\mathrm{Fe}-\mathrm{As}_{3}, \alpha(\mathrm{deg})$ & $113.03(3)$ \\
\hline$S_{3}(\AA)$ & $1.697(2)$ \\
\hline$S_{1}(\AA)$ Fig. 3(a) & $2.358(2)$ \\
\hline$h_{\mathrm{pn}}\left(S_{2} / 2\right)(\AA)$ & $1.293(2)$ \\
\hline Calculated density $\left(\mathrm{g} / \mathrm{cm}^{3}\right)$ & 7.297 \\
\hline Absorption coefficient $\left(\mathrm{mm}^{-1}\right)$ & 35.630 \\
\hline$F(000)$ & 244 \\
\hline Crystal size, $\left(\mu \mathrm{m}^{3}\right)$ & $117 \times 77 \times 18$ \\
\hline$\theta$ range for data collection & $2.44^{\circ}-48.39^{\circ}$ \\
\hline Index ranges & $7 \leqslant h \leqslant 7,-8 \leqslant k \leqslant 7,-16 \leqslant l \leqslant 17$ \\
\hline Reflections collected/unique & $3673 / 409 R_{\text {int. }}=0.0536$ \\
\hline Completeness to $2 \theta$ & $98.3 \%$ \\
\hline Data/restraints/parameters & $409 / 0 / 13$ \\
\hline Goodness of fit on $F^{2}$ & 1.135 \\
\hline Final $R$ indices $[I 2 \sigma(I)]$ & $R_{1}=0.0324, \mathrm{w} R_{2}=0.0742$ \\
\hline$R$ indices (all data) & $R_{1}=0.0410, \mathrm{w} R_{2}=0.0767$ \\
\hline$\Delta \rho_{\max }$ and $\Delta \rho_{\min }\left(\mathrm{e} / \mathrm{A}^{3}\right)$ & 4.238 and -4.408 \\
\hline
\end{tabular}




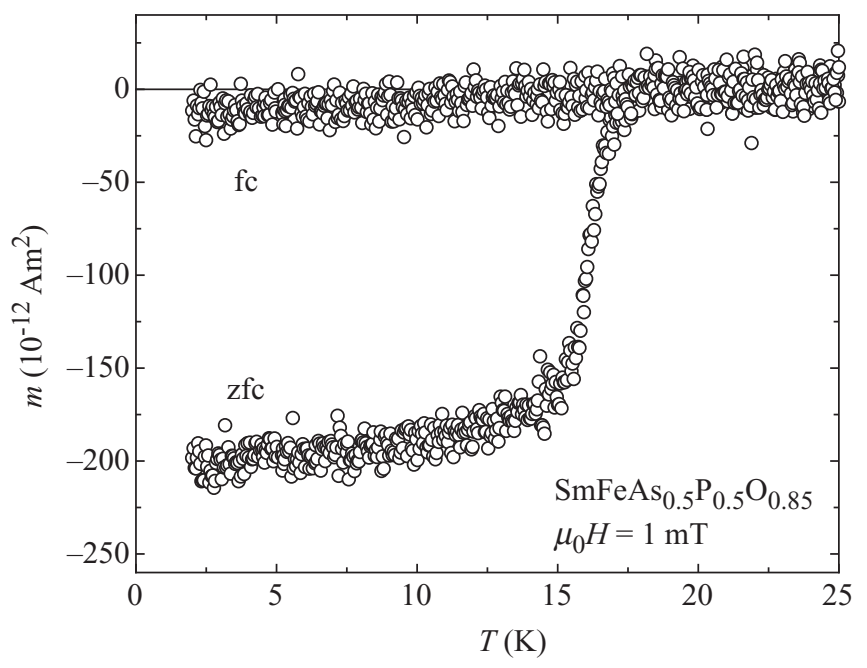

FIG. 10. The temperature dependence (zero-field-cooled and field-cooled) of the magnetic moment of a $\mathrm{SmFeAs}_{0.5} \mathrm{P}_{0.5} \mathrm{O}_{0.85}$ (nominal composition) single crystal in a magnetic field of $1 \mathrm{mT}$ applied parallel to the $c$-axis.

one, with $2 \Delta / \mathrm{k}_{\mathrm{B}} T_{\mathrm{c}}$ ratios of about 4 (close to the BCS value) and 13 , respectively. Although the ratio for the large gap is very high and has still to be fully understood, the two-gap model fits the data quite well, while the single-gap one disregards a large portion of the experimental curve and would give an anomalously high $2 \Delta / \mathrm{k}_{\mathrm{B}} T_{\mathrm{c}}$ (from about 6 to 8 ) for a one-gap picture.

The Andreev-reflection features gradually decrease in amplitude with increasing temperature, until they completely disappear close to the critical temperature and the two-band model can follow the evolution of the curves with increasing temperature reasonably well. The behavior of the two gaps, as obtained from the fit, is shown in the inset to Fig. 9 (symbols): The small gap follows a BCS trend rather well (red solid line), while the large one apparently decreases almost linearly above $\sim T_{\mathrm{c}} / 2$. In the same range, however, the uncertainty in the large gap is rather big, so that a BCS trend (blue solid line) for this gap cannot be ruled out.

These results seem to indicate the presence of two nodeless gaps in the $\mathrm{SmFeAs}_{0.5} \mathrm{P}_{0.5} \mathrm{O}$ compound, in agreement with results obtained by PCAR spectroscopy measurements on other Fe-based superconductors of the 1111, ${ }^{40,41}$ but also of the 122 family. ${ }^{39,45}$ In particular, nodeless superconductivity in this compound is also expected according to the theoretical work by Kuroki et al ${ }^{46}$ : Given the lattice constants $[a=$ 3.90664(2) $\AA ; c=8.3489(4) \AA]$ and the pnictogen height $\left[h_{\mathrm{Pn}}=1.319(8) \AA\right]$, by looking at the upper panel of Fig. 19 in Ref. 46, it is possible to observe that our $\mathrm{SmFeAs}_{0.5} \mathrm{P}_{0.5} \mathrm{O}$ lies in the "high $T_{\mathrm{c}}$, nodeless" region. In this regard, our results may support the picture given in Ref. 46, i.e., that lattice structure considerably affects the Fermi surface and, as a consequence, the gap functions in these compounds.

\section{F. Magnetic and transport properties of single crystals}

For the measurements presented here, we chose single crystals from the batches with $\mathrm{SmFeAs}_{0.5} \mathrm{P}_{0.5} \mathrm{O}$ and $\mathrm{SmFeAs}_{0.5} \mathrm{P}_{0.5} \mathrm{O}_{0.85}$ nominal compositions. According to $\mathrm{XRD}$, they are free of impurities, twins, or intergrowing crystals and show well-resolved reflection patterns, indicating high-quality perfection. As a representative example, the structural refinement parameters for $\mathrm{SmFeAs}_{0.5} \mathrm{P}_{0.5} \mathrm{O}_{0.85}$ single crystal with $T_{\mathrm{c}}=17 \mathrm{~K}$ are summarized in Table II.

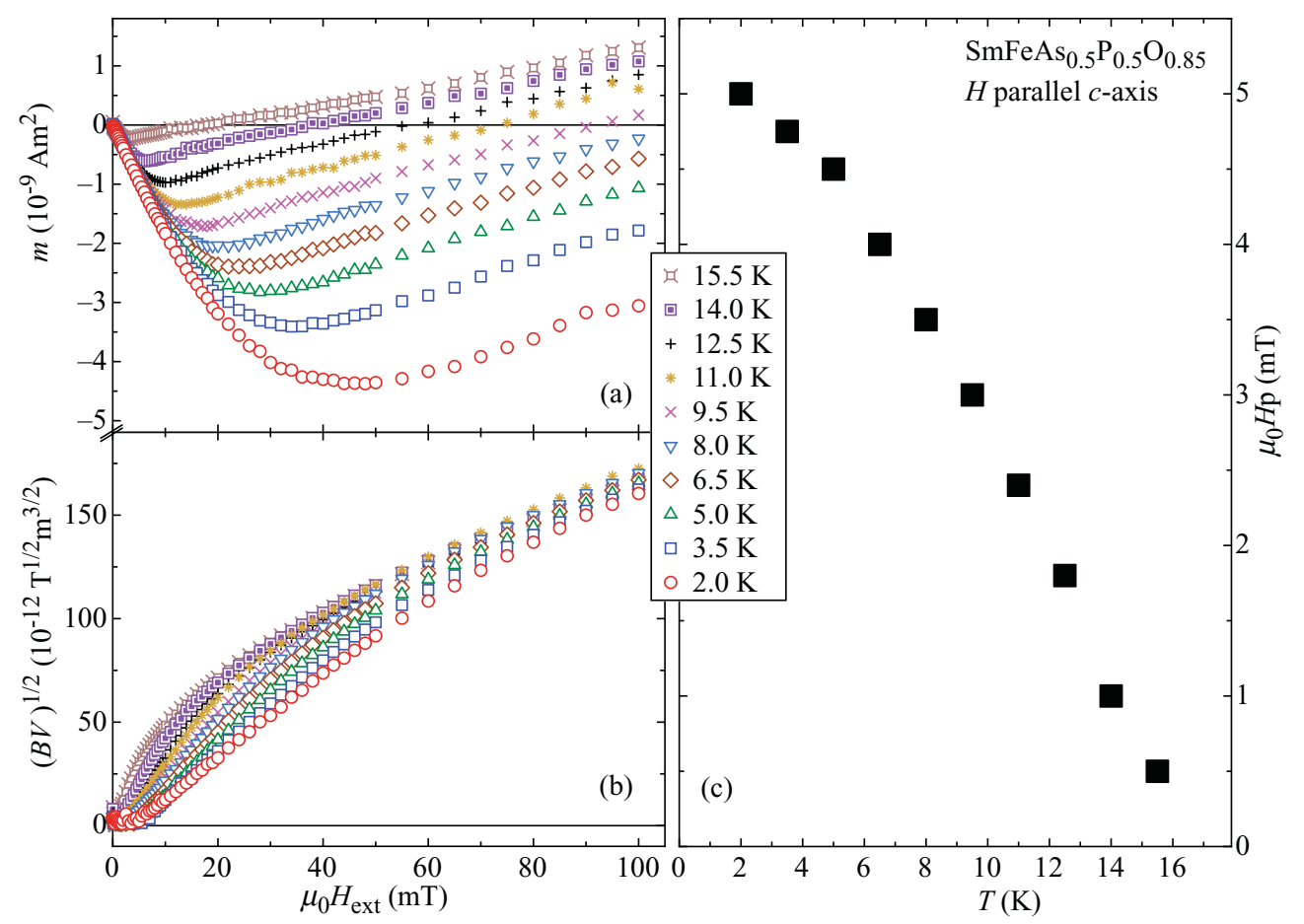

FIG. 11. (Color online) (a) Initial magnetization curves of a $\operatorname{SmFeAs}{ }_{0.5} \mathrm{P}_{0.5} \mathrm{O}_{0.85}$ crystal recorded in the temperature range between 2 and $17 \mathrm{~K}$. (b) The quantity $(B V)^{1 / 2}$ calculated from the magnetic moment and plotted as a function of magnetic field. (c) The temperature evolution of the first penetration field $H_{\mathrm{p}}$. The estimate $\mu_{0} H_{\mathrm{p}}(0) \approx 5 \mathrm{mT}$ yields an in-plane magnetic penetration depth $\lambda_{a b}(0) \approx 200 \mathrm{~nm}$. 
Figure 10 shows the temperature dependence of the magnetic moment of a SmFeAs ${ }_{0.5} \mathrm{P}_{0.5} \mathrm{O}_{0.85}$ single crystal, measured while increasing the temperature in a magnetic field of $1 \mathrm{mT}$ after zero-field-cooling (zfc) and field-cooling (fc). The direction of the applied field was in all magnetic measurements along the crystallographic $c$-axis. The single crystal studied was of nearly rectangular shape, obeying the approximate dimensions of $80 \times 60 \times 5 \mu \mathrm{m}^{3}$. The observed signal at lowest temperature is indicative for bulk superconductivity, and the sharp transition for a small doping spread in the sample. The onset is estimated to be $T_{\mathrm{c}} \sim 17 \mathrm{~K}$.

Initial magnetization curves were recorded for the $\mathrm{SmFeAs}_{0.5} \mathrm{P}_{0.5} \mathrm{O}_{0.85}$ single crystal in the temperature range between 2 and $17 \mathrm{~K}$. These allowed us to gain information on the first penetration field $H_{\mathrm{p}}$, which denotes the magnetic field above which vortices enter the sample. Figure 11(a) presents the measured magnetic moment in low magnetic fields, which show the linear decrease of $m(H)$ in the Meissner state and the following upturn in magnetization due to the entrance of vortices into the bulk. The field $H_{\mathrm{p}}$ was estimated according to the procedure discussed in Ref. 47 . The quantity $(B V)^{1 / 2}$ was calculated from the measured magnetic moment and plotted as a function of magnetic field [see Fig. 11(b)]. Here, $B$ denotes the magnetic induction, and $V$ is the sample volume. Since $B=\mu_{0}(M+H)=\mu_{0}(m / V+H)=0$ in the Meissner state, it is possible to calculate, from the data of $m(H)$, the field $H_{\mathrm{p}}$ above which this equality is invalid. Using knowledge of $m$ and $H$, we calculate the quantity $B V$, which empirically scales as the square root of $H$ above $H_{\mathrm{p}}$. Hence, a plot of $(B V)^{1 / 2}$ as a function of $H$ allows a straightforward determination of $H_{p}$. The sudden increase from zero occurs due to the penetration of vortices at $H_{p}$. In Fig. 11(c), we show the temperature evolution for $H_{\mathrm{p}}$ for the $\mathrm{SmFeAs}_{0.5} \mathrm{P}_{0.5} \mathrm{O}_{0.85}$ single crystal as extracted from Fig. 11(b). The field $H_{\mathrm{p}}$ is related to the intrinsic $H_{\mathrm{cl}}$, but it is systematically lowered as compared to the latter due to the reduction of the Meissner state by the shape factor of the plate-like single crystal, which unfortunately is expected to contribute significantly. Approaching the base temperature, $\mu_{0} H_{\mathrm{p}}(0) \approx 5 \mathrm{mT}$, which yields an estimate for the in-plane magnetic penetration depth $\lambda_{a b}(0) \approx 200 \mathrm{~nm}$.

The crystals with various $T_{\mathrm{c}}$ were used for magnetotransport studies. Figure 12(a) shows the temperature dependence of the magnetoresistance from $2 \mathrm{~K}$ to $300 \mathrm{~K}$
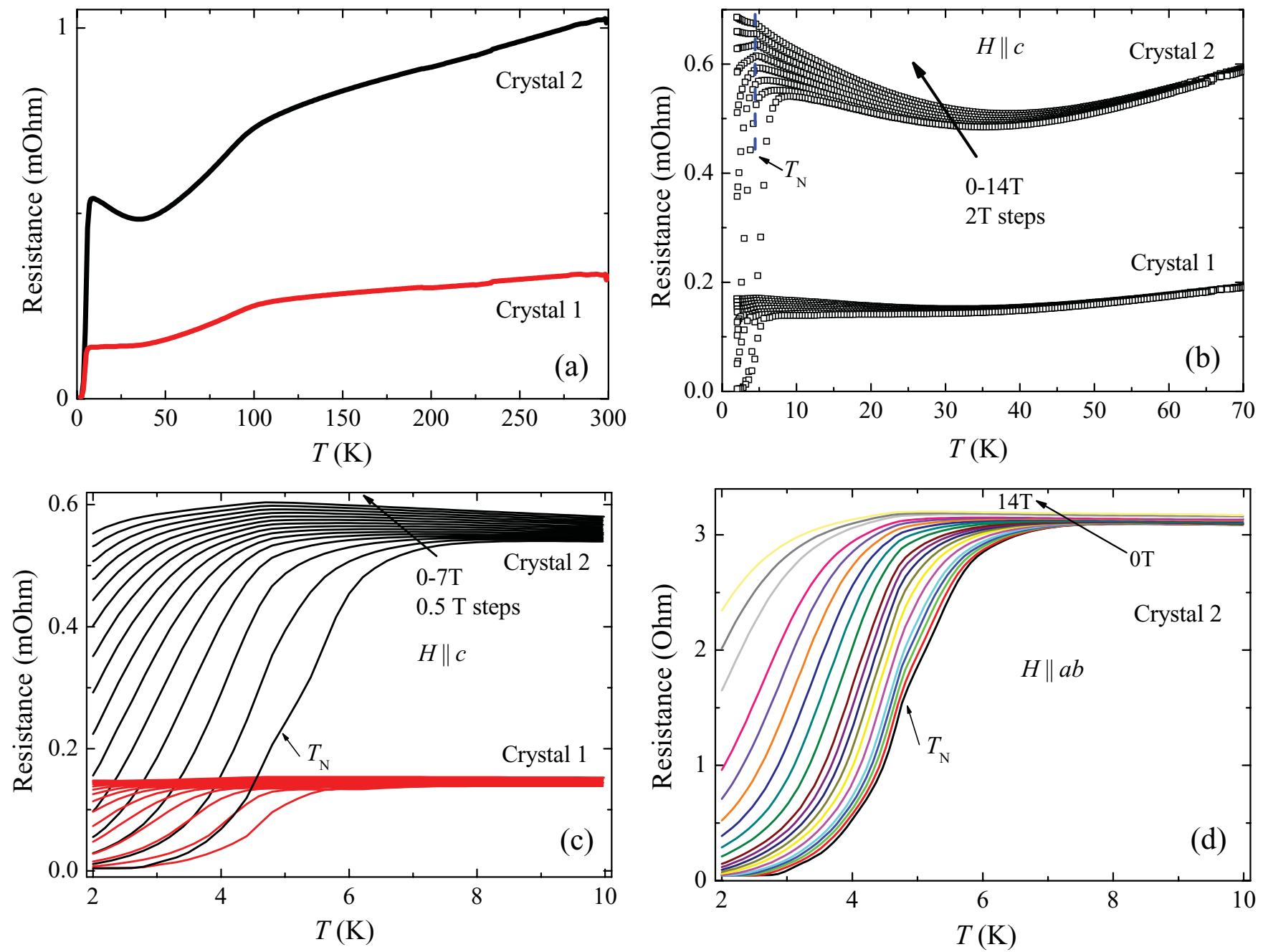

FIG. 12. (Color online) Temperature dependence of the resistance, in various fields, applied parallel to the $\mathrm{Fe}_{2}(\mathrm{As}, \mathrm{P})_{2}$ layers $(H \| a b)$ and perpendicular to them $(H \| c)$ for two $\mathrm{SmFeAs}_{0.5} \mathrm{P}_{0.5} \mathrm{O}$ (nominal composition) crystals grown under high pressure. The dashed line and arrows indicate the magnetic ordering temperature of $\mathrm{Sm}$ at $\sim 4.8 \mathrm{~K}$. 
for two $\mathrm{SmFeAs}_{0.5} \mathrm{P}_{0.5} \mathrm{O}$ crystals. For the parent $\mathrm{SmFeAsO}$ compound, it is well known that there is a drop in resistivity around $135 \mathrm{~K}$ due to a structural transition associated with a SDW-type antiferromagnetic order. As we can see in Fig. 12(a), upon P substitution, the anomaly in the resistance becomes less obvious and shifts to lower temperatures. For one crystal, $\rho(\mathrm{T})$ shows a strong upturn at low temperature, which could be the result of charge carrier localization due to the disorder in the conducting layers as $\mathrm{P}$ is substituted on the As site. In both samples, an onset critical temperature $T_{\mathrm{c} \text {,on }}$ of $\sim 5.8 \mathrm{~K}$ and $\sim 8 \mathrm{~K}$ is observed. Figure $12(\mathrm{~b})$ and 12 (c) shows the temperature dependencies of the magnetoresistance in various magnetic fields applied parallel to the $\mathrm{Fe}_{2}(\mathrm{As}, \mathrm{P})_{2}$ layers $(H \| a b)$ and perpendicular to them $(H \|$ $c)$. Furthermore, the magnetoresistance shows that magnetic moments of $\mathrm{Sm}^{3+}$ ions order antiferromagnetically below magnetic transition of $\sim 4.8 \mathrm{~K}$ [dashed line in Fig. 12(b) and arrows in Figs. 12(c) and 12(d)]. By applying higher fields, the visibility of $T_{\mathrm{N}}$ in Figs. 12(c) and 12(d) is not so obvious due to broadening of the superconducting transition. As we recently reported ${ }^{48}$ the complete suppression of $\mathrm{Sm}$ antiferromagnetism would require fields in excess of $60 \mathrm{~T}$. While structure analysis attests only one overall structure, we nevertheless attribute the two electronic states, i.e., SDW-type antiferromagnetic order and superconductivity, to microscopic electronic inhomogeneity, but this requires experimental verification.

As one representative example of magnetoresistance data obtained on the crystals from the $\mathrm{SmFeAs}_{0.5} \mathrm{P}_{0.5} \mathrm{O}_{0.85}$ batches, in Fig. 13 we present the data for a single crystal with $T_{\mathrm{c}, \text { zero }}=$ $21.7 \mathrm{~K}$. Magnetoresistance measurements, $\rho(T, H)$, near $T_{\mathrm{c}}$ for magnetic fields parallel $(H \| a b)$ and perpendicular $(H \|$ c) to the FeAs/P planes show remarkably different behavior than those of Sm 1111 substituted with $\mathrm{F}$ for $\mathrm{O}$ or Th for Sm. ${ }^{17,24}$ In the latter two cases, the magnetic fields cause only a slight shift of the onset of superconductivity, but a significant broadening of the transition, indicating weak pinning and accordingly large flux flow dissipation. In P-substituted Sm 1111 crystals, the presence of magnetic fields shifts the onset of superconductivity to lower temperatures but does not cause a significant broadening of the transition. The upper critical fields $H_{\mathrm{c} 2} \| a b$ and $H_{\mathrm{c} 2} \| c$ extracted from the resistivity measurements are shown in Fig. 13(b). The upper critical field $H_{\mathrm{c} 2}$ in $\mathrm{SmFeAs}_{0.5} \mathrm{P}_{0.5} \mathrm{O}_{0.85}$ increase linearly with decreasing temperature $\sim 1 \mathrm{~K}$ below $T_{\mathrm{c}}$, with a slope of $\sim 5.7 \mathrm{~T} / \mathrm{K}$ $(H \| a b)$ and $\sim 1.3 \mathrm{~T} / \mathrm{K}(H \| c)$. The slopes in Sm 1111 crystals substituted with $\mathrm{F}$ or Th depend strongly on the criterion due to pronounced broadening. We found 8.0-5.5 T/K for $H \|$ $a b$ and $3.3-1.2 \mathrm{~T} / \mathrm{K}$ for $H \| c .^{15,23}$ All these large slopes indicate very high values of $H_{\mathrm{c} 2}(0)$. The $\mathrm{Sm} 1111$ structure is more anisotropic than the structure of 122 , which is already manifested in the upper critical field anisotropy $\gamma_{H}=H_{\mathrm{c} 2}$ $\left\|a b / H_{\mathrm{c} 2}\right\| c .{ }^{15}$ The data presented in Fig. 13(b) suggest that the anisotropy $\gamma_{H}$ in $\operatorname{SmFeAs}_{0.5} \mathrm{P}_{0.5} \mathrm{O}_{0.85}$ sufficiently below $T_{\mathrm{c}}$ decreases from $\sim 7.3$ to $\sim 6.6$ with decreasing temperature. Such temperature-dependent $\gamma_{H}$ has already been observed in the $\mathrm{SmFeAsO}_{0.8} \mathrm{~F}_{0.2}$ and $\mathrm{NdFeAsO}_{0.8} \mathrm{~F}_{0.2}$ single crystals, ${ }^{17,49}$ and this further supports a multiband superconductivity scenario, where different parts of the Fermi surface sheet develop distinct gaps in the superconducting
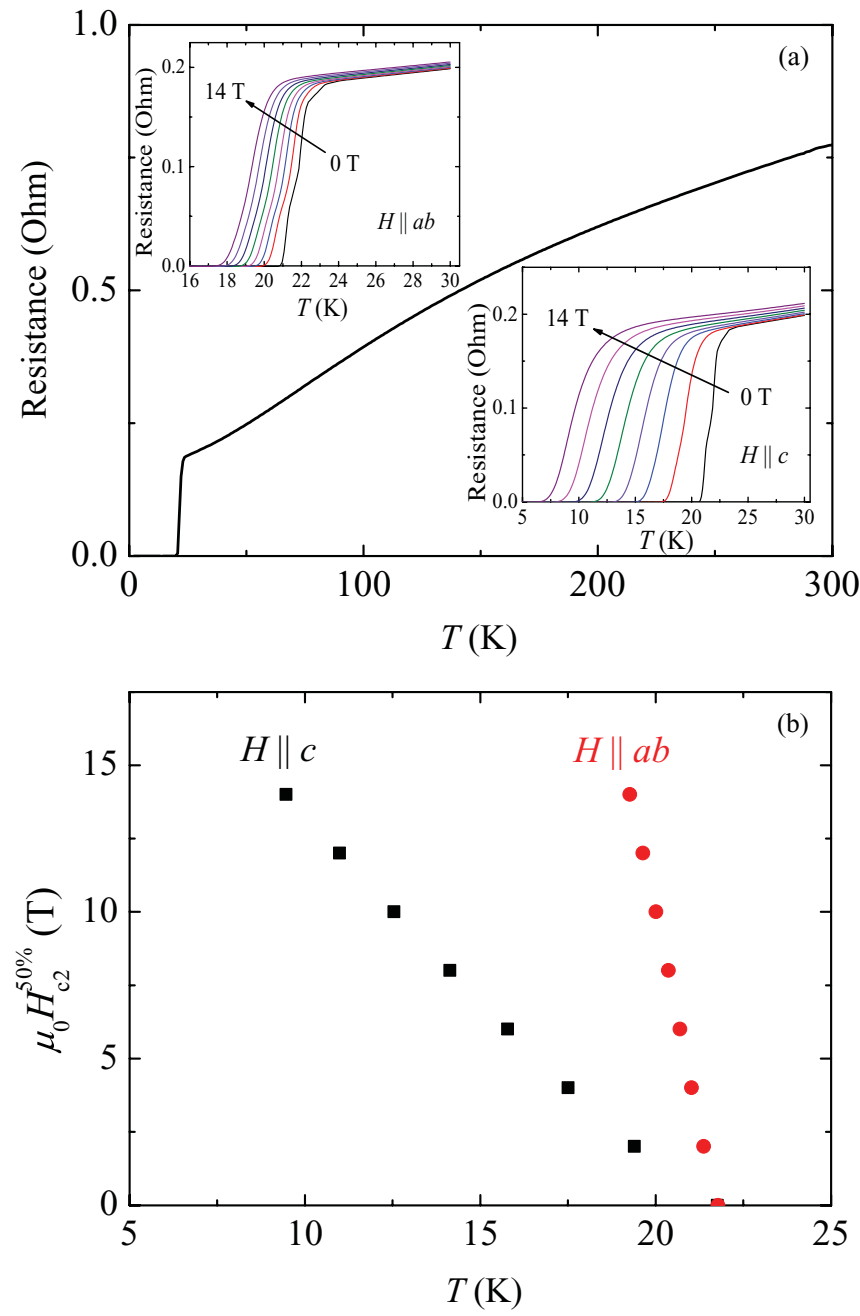

FIG. 13. (Color online) (a) Temperature dependence of the resistance for a $\mathrm{SmFeAs}{ }_{0.5} \mathrm{P}_{0.5} \mathrm{O}_{0.85}$ (nominal composition) single crystal. Left and right insets show measurements with the field applied parallel to the $\mathrm{Fe}_{2}(\mathrm{As}, \mathrm{P})_{2}$ layers $(H \| a b)$ and perpendicular to them $(H \| c)$, in various magnetic fields $(0,2,4,6,8,10,12,14 \mathrm{~T})$. (b) Temperature dependence of the upper critical field with $H \| a b$ and $H \| c$. To determine $H_{\mathrm{c} 2}$, the $50 \% \rho_{\mathrm{n}}$ criterion was used.

state. However, in order to clarify its origin, more systematic experimental work is required.

\section{CONCLUSIONS}

Polycrystalline and single-crystalline samples of $\mathrm{SmFeAs}_{1-x} \mathrm{P}_{x} \mathrm{O}$ were successfully prepared using the quartz ampoule and the high-pressure cubic anvil techniques. In the case of ambient pressure-prepared samples, substitution of As by $\mathrm{P}$ results in a decrease in the unit-cell volume and a continuous suppression of both the ordering temperature and the frequency of magnetic order. The appearance of superconductivity in the $\mathrm{SmFeAs}_{1-x} \mathrm{P}_{x} \mathrm{O}_{1-y}$ samples caused by oxygen deficiency was realized only after heat treatment under high pressure. The pressure-induced oxygen deficiency brings the $\mathrm{Sm}-\mathrm{O}$ charge-transfer layer closer to the superconducting As/P-Fe-As/P block and facilitates 
electron transfer. For superconducting $\mathrm{SmFeAs}_{0.5} \mathrm{P}_{0.5} \mathrm{O}_{1-y}$ samples, only dynamic magnetism survives, while the ambient-prepared samples with the same amount of $\mathrm{P}$ substitution still show a static magnetic moment at temperatures below $\sim 60 \mathrm{~K}$. Point-contact Andreev-reflection spectroscopy indicates the existence of two energy gaps in superconducting samples, supporting a common multigap scenario proposed for FeAs-based superconductors. Single crystals of $\mathrm{SmFeAs}_{1-x} \mathrm{P}_{x} \mathrm{O}_{1-y}$ have been grown under high pressure, and their crystallographic and basic superconducting state properties were presented. The upper critical field deduced from resistance measurements is anisotropic with slopes of $\sim 5.7 \mathrm{~T} / \mathrm{K}(H \| a b$ plane $)$ and $\sim 1.3 \mathrm{~T} / \mathrm{K}(H \| c$-axis $)$ sufficiently far below $T_{\mathrm{c}}$.

\section{ACKNOWLEDGMENTS}

We would like to thank P. Wägli for the EDX analysis. This work was supported by the Swiss National Science Foundation and the National Center of Competence in Research MaNEP (Materials with Novel Electronic Properties), Project No. 124616. *zhigadlo@phys.ethz.ch

${ }^{1}$ Y. Kamihara, H. Hiramatsu, M. Hirano, R. Kawamura, H. Yanagi, T. Kamiya, and H. Hosono, J. Am. Chem. Soc. 128, 10012 (2006).

${ }^{2}$ Y. Kamihara, T. Watanabe, M. Hirano, and H. Hosono, J. Am. Chem. Soc. 130, 3296 (2008).

${ }^{3}$ K. Ishida, Y. Nakai, and H. Hosono, J. Phys. Soc. Jpn. 78, 062001 (2009).

${ }^{4}$ For recent reviews, see D. C. Johnston, Adv. Phys. 59, 803 (2010); J.-P. Paglione, and R. L. Greene, Nat. Phys. 6, 645 (2010); P. C. Canfield, and S. L. Bud'ko, Annu. Rev. Condens. Mat. Phys. 1, 27 (2010).

${ }^{5}$ J. Day, Q. Si, J.-X. Zhu, and E. Abrahams, Proc. Natl. Acad. Sci. USA 106, 4118 (2009).

${ }^{6}$ C. Wang, S. Jiang, Q. Tao, Z. Ren, Y. Li, L. Li, C. Feng, J. Dai, G. Cao, and Z.-A. Xu, EPL 86, 47002 (2009).

${ }^{7}$ S. Jiang, H. Xing, G. Xuan, C. Wang, Z. Ren, C. Feng, J. Dai, Z.-A. Xu, and G. Cao, J. Phys. Condens. Mat. 21, 382203 (2009).

${ }^{8}$ Z. Ren, Q. Tao, S. Jiang, C. Feng, C. Wang, J. Dai, G. Cao, and Z.-A. Xu, Phys. Rev. Lett. 102, 137002 (2009).

${ }^{9}$ H. L. Shi, H. X. Yang, H. F. Tian, J. B. Lu, Z. W. Wang, Y. B. Qin, Y. J. Song, and J. Q. Li, J. Phys. Condens. Mat. 22, 125702 (2010).

${ }^{10}$ C. de la Cruz, W. Z. Hu, S. Li, Q. Huang, J. W. Lynn, M. A. Green, G. F. Chen, N. L. Wang, H. A. Mook, Q. Si, and P. Dai, Phys. Rev. Lett. 104, 017204 (2010).

${ }^{11}$ W. Schnelle, A. Leithe-Jasper, R. Gumeniuk, U. Burkhardt, D. Kashinathan, and H. Rosner, Phys. Rev. B 79, 214516 (2009); S. Sharma, A. Bharathi, S. Chandra, V. R. Reddy, S. Paulraj, A. T. Satya, V. S. Sastry, A. Gupta, and C. S. Sundar, ibid. 81, 174512 (2010).

${ }^{12}$ M. A. McGuire, D. J. Singh, A. S. Sefat, B. C. Sales, and D. Mandrus, J. Solid State Chem. 182, 2326 (2009).

${ }^{13}$ T. M. McQueen, M. Regulacio, A. J. Williams, Q. Huang, J. W. Lynn, Y. S. Hor, D. V. West, M. A. Green, and R. J. Cava, Phys. Rev. B 78, 024521 (2008).

${ }^{14}$ Y. Kamihara, H. Hiramatsu, M. Hirano, Y. Kobayashi, S. Kitao, S. Higashitaniguchi, Y. Yoda, M. Seto, and H. Hosono, Phys. Rev. B 78, 184512 (2008).

${ }^{15}$ Y. K. Li, X. A. Lin, T. Zhou, J. Q. Shen, Y. K. Luo, Q. A. Tao, G. H. Cao, and Z. A. Xu, Physica C 470, S493 (2010).

${ }^{16}$ N. D. Zhigadlo, S. Katrych, Z. Bukowski, S. Weyeneth, R. Puzniak, and J. Karpinski, J. Phys. Condens. Mat. 20, 342202 (2008).

${ }^{17}$ J. Karpinski, N. D. Zhigadlo, S. Katrych, Z. Bukowski, P. Moll, S. Weyeneth, H. Keller, R. Puzniak, M. Tortello, D. Daghero,
R. Gonnelli, I. Maggio-Aprile, Y. Fasano, Ø. Fischer, K. Rogacki, and B. Batlogg, Physica C 469, 370 (2009).

${ }^{18}$ J. Rodrígues-Carvajal, Physica B 192, 55 (1993).

${ }^{19}$ APEX 2 Version 2009.9 (Bruker AXS Inc.); SAINT Version 7.68 A (Bruker AXS Inc., 2009); SADABS Version 2008/1 (Sheldrick, Bruker AXS Inc.)

${ }^{20} \mathrm{G}$. Sheldrick, SHELXS-97, Program for the Solution of Crystal Structures (University of Göttingen, Germany, 1997); SHELXL-97, Program for the Refinement of Crystal Structures (University of Göttingen, Germany, 1997).

${ }^{21}$ P. J. W. Moll, R. Puzniak, F. Balakirev, K. Rogacki, J. Karpinski, N. D. Zhigadlo, and B. Batlogg, Nat. Mater. 9, 628 (2010).

${ }^{22}$ R. S. Gonnelli, D. Daghero, D. Delaude, M. Tortello, G. A. Ummarino, V. A. Stepanov, J. S. Kim, R. K. Kremer, A. Sanna, G. Profeta, and S. Massidda, Phys. Rev. Lett. 100, 207004 (2008).

${ }^{23}$ P. Fischer, G. Frey, M. Koch, M. Könnecke, V. Pomjakushin, J. Schefer, R. Thut, N. Schlumpf, R. Bürge, U. Greuter, S. Bondt, and E. Berruyer, Physica B 276-278, 146 (2000).

${ }^{24}$ N. D. Zhigadlo, S. Katrych, S. Weyeneth, R. Puzniak, P. J. W. Moll, Z. Bukowski, J. Karpinski, H. Keller, and B. Batlogg, Phys. Rev. B 82, 064517 (2010)

${ }^{25}$ A. Ricci, N. Poccia, B. Joseph, L. Barba, G. Arrighetti, G. Ciasca, J.-Q. Yan, R. W. McCallum, T. A. Lograsso, N. D. Zhigadlo, J. Karpinski, and A. Bianconi, Phys. Rev. B 82, 144507 (2010); T. Mertelj, P. Kusar, V. V. Kabanov, L. Stojchevska, N. D. Zhigadlo, S. Katrych, Z. Bukowski, J. Karpinski, S. Weyeneth, and D. Mihailovic, ibid. 81, 224504 (2010); M. Matusiak, T. Plackowski, Z. Bukowski, N. D. Zhigadlo, and J. Karpinski, ibid. 79, 212502 (2009); M. Le Tacon, T. R. Forrest, Ch. Rüegg, A. Bosak, A. C. Walters, R. Mittal, H. M. Rønnow, N. D. Zhigadlo, S. Katrych, J. Karpinski, J. P. Hill, M. Krisch, and D. F. McMorrow, ibid. 80, 220504(R) (2009).

${ }^{26}$ R. Khasanov, S. Sanna, G. Prando, Z. Shermadini, M. Bendele, A. Amato, P. Carretta, R. De Renzi, J. Karpinski, S. Katrych, H. Luetkens, and N. D. Zhigadlo, Phys. Rev. B 84, 100501(R) (2011).

${ }^{27}$ R. D. Shannon, Acta Crystallogr. Sect. A: Cryst. Phys., Diffr., Theor. Gen. Crystallogr. 32, 751 (1976).

${ }^{28}$ C.-H. Lee, A. Iyo, H. Eisaki, H. Kito, M. T. Fernandez-Diaz, T. Ito, K. Kihou, H. Matsuhata, M. Braden, and K. Yamada, J. Phys. Soc. Jpn. 77, 083704 (2008).

${ }^{29}$ R. Hoffman, and C. Zheng, J. Phys. Chem. 89, 4175 (1985); D. Johrendt, C. Felser, O. Jepsen, O. K. Andersen, A. Mewis, and J. Rouxel, J. Solid State Chem. 130, 254 (1997). 
${ }^{30}$ S. A. Jia, P. Jiramongkolchai, M. R. Suchomel, B. H. Toby, J. G. Checkelsky, N. P. Ong, and R. J. Cava, Nat. Phys. 7, 207 (2011); S. A. Jia, A. J. Williams, P. W. Stephens, and R. J. Cava, Phys. Rev. B 80, 165107 (2009).

${ }^{31}$ A. Amato, Rev. Mod. Phys. 69, 1119 (1997).

${ }^{32}$ A. T. Savici, Y. Fudamoto, I. M. Gat, T. Ito, M. I. Larkin, Y. J. Uemura, G. M. Luke, K. M. Kojima, Y. S. Lee, M. A. Kastner, R. J. Birgeneau, and K. Yamada, Phys. Rev. B 66, 014524 (2002).

${ }^{33}$ A. J. Drew, Ch. Niedermayer, P. J. Baker, F. L. Pratt, S. J. Blundell, T. Lancaster, R. H. Liu, G. Wu, X. H. Chen, I. Watanabe, V. K. Malik, A. Dubroka, M. Rössle, K. W. Kim, C. Baines, and C. Bernhard, Nat. Mater. 8, 310 (2009).

${ }^{34}$ H. Maeter, H. Luetkens, Yu. G. Pashkevich, A. Kwadrin, R. Khasanov, A. Amato, A. A. Gusev, K. V. Lamonova, D. A. Chervinskii, R. Klingeler, C. Hess, G. Behr, B. Büchner, and H.-H. Klauss, Phys. Rev. B 80, 094524 (2009).

${ }^{35}$ H.-H. Klauss, H. Luetkens, R. Klingeler, C. Hess, F. J. Litterst, M. Kraken, M. M. Korshunov, I. Eremin, S.-L. Drechsler, R. Khasanov, A. Amato, J. Hamann-Borrero, N. Leps, A. Kondrat, G. Behr, J. Werner, and B. Büchner, Phys. Rev. Lett. 101, 077005 (2008).

${ }^{36}$ R. Khasanov, H. Luetkens, A. Amato, H.-H. Klauss, Z.-A. Ren, J. Yang, W. Lu, and Z.-X. Zhao, Phys. Rev. B 78, 092506 (2008).

${ }^{37}$ A. J. Drew, F. L. Pratt, T. Lancaster, S. J. Blundell, P. J. Baker, R. H. Liu, G. Wu, X. H. Chen, I. Watanabe, V. K. Malik, A. Dubroka, K. W. Kim, M. Rössle, and C. Bernhard, Phys. Rev. Lett. 101, 097010 (2008).

${ }^{38}$ L. Ding, C. He, J. K. Dong, T. Wu, R. H. Liu, X. H. Chen, and S. Y. Li, Phys. Rev. B 77, 180510(R) (2008).

${ }^{39}$ M. Tortello, D. Daghero, G. A. Ummarino, V. A. Stepanov, J. Jiang, J. D. Weiss, E. E. Hellstrom, and R. S. Gonnelli, Phys. Rev. Lett. 105, 237002 (2010).
${ }^{40}$ D. Daghero, M. Tortello, R. S. Gonnelli, V. A. Stepanov, N. D. Zhigadlo, and J. Karpinski, Phys. Rev. B 80, 060502 (R) (2009).

${ }^{41}$ R. S. Gonnelli, D. Daghero, M. Tortello, G. A. Ummarino, V. A. Stepanov, J. S. Kim, and R. K. Kremer, Phys. Rev. B 79, 184526 (2009).

${ }^{42}$ A. Pleceník, M. Grajcar, Š. Beňačka, P. Seidel, and A. Pfuch, Phys. Rev. B 49, 10016 (1994).

${ }^{43}$ G. E. Blonder, M. Tinkham, and T. M. Klapwijk, Phys. Rev. B 25, 4515 (1982).

${ }^{44}$ S. Kashiwaya, Y. Tanaka, M. Koyanagi, and K. Kajimura, Phys. Rev. B 53, 2667 (1996).

${ }^{45}$ P. Szabó, Z. Pribulová, G. Pristáš, S. L. Bud'ko, P. C. Canfield, and P. Samuely, Phys. Rev. B 79, 012503 (2009).

${ }^{46}$ K. Kuroki, H. Usui, S. Onari, R. Arita, and H. Aoki, Phys. Rev. B 79, 224511 (2009).

${ }^{47}$ M. Bendele, S. Weyeneth, R. Puzniak, A. Maisuradze, E. Pomjakushina, K. Conder, V. Pomjakushin, H. Luetkens, S. Katrych, A. Wisniewski, R. Khasanov, and H. Keller, Phys. Rev. B 81, 224520 (2010).

${ }^{48}$ S. Weyeneth, P. J. W. Moll, R. Puzniak, K. Ninios, F. F. Balakirev, R. D. McDonald, H. B. Chan, N. D. Zhigadlo, S. Katrych, Z. Bukowski, J. Karpinski, H. Keller, B. Batlogg, and L. Balicas, Phys. Rev. B 83, 134503 (2011).

${ }^{49}$ S. Weyeneth, R. Puzniak, N. D. Zhigadlo, S. Katrych, Z. Bukowski, J. Karpinski, and H. Keller, J. Supercond. Nov. Magn. 22, 347 (2009).

${ }^{50}$ Y. Mizuguchi, Y. Hara, K. Deguchi, S. Tsuda, T. Yamaguchi, K. Takeda, H. Kotegawa, H. Tou, and Y. Takano, Supercond. Sci. Technol. 23, 054013 (2010), and references therein. 